\title{
Synchronization and Dynamic Consensus of Heterogeneous Networked Systems
}

\author{
Elena Panteley Antonio Loría
}

\begin{abstract}
We present an analysis framework for the study of synchronization of heterogeneous nonlinear systems interconnected over networks described by directed graphs. Heterogeneous systems may have totally different dynamical models, albeit of the same dimension, or may possess equal models with different lumped parameters. We show that their behavior, when network-interconnected, is fully characterized in terms of two properties whose study may be recasted in terms of the stability analysis of two corresponding interconnected dynamical systems that evolve in orthogonal spaces: on one hand, we have the so-called emergent dynamics and, on the other, the synchronization error dynamics. Based on this premise, we introduce the concept of dynamic consensus and we present results on robust stability which assess the conditions for practical asymptotic synchronization of networked systems and characterize their collective behavior. To illustrate our main theoretical findings we broach a brief case-study on mutual synchronization of heterogeneous chaotic oscillators.
\end{abstract}

\section{INTRODUCTION}

In the context of network-interconnected systems, synchronization corresponds to the case in which all the systems asymptotically follow the same trajectory, up to a certain "shift"1. Understanding this phenomenon and describing it with certain mathematical rigor has been an active research field during the last decades in a variety of generic disciplines including biology, sociology, physics, computer science, telecommunications, statistics and dynamical systems. Beyond these, new interdisciplinary research fields, such as synergetics and the study of self-organized systems, focus on distinct principles that govern the behavior of complex interconnected systems -see [2], [3]. In the control community, the study of synchronization and consensus also continues to attract an increasing interest - see, for instance, [4]-[5].

From a dynamical-systems viewpoint, synchronization depends on some key factors which include:

- the type and strength of the interconnection among the nodes,

- the network structure,

- the dynamics of the individual units.

In addition to this, the local interactions among the initially disordered systems generate a form of global coordination which is referred to as the mean field behavior.

In general, the nodes' interconnections depend on the strength of the coupling and on output functions of the nodes' state variables. The interaction is also determined by the form of coupling. This may be linear, as it is most commonly

The authors are with the CNRS, France, at Laboratoire des sig naux et systèmes, CentraleSuplec 91192 Gif-sur-Yvette, France. E-mail: panteleyelss.supelec.fr. The first author is also with ITMO University, Kronverkskiy av. 49, Saint Petersburg, 197101, Russia.

${ }^{1}$ See [1] for different definitions of synchronization. assumed, but it may also be nonlinear, as in the well-known example of Kuramoto's oscillator model in which case the interconnection is established via sinusoids -see [11], [12], [13]. Other types of nonlinear coupling appear, for instance, in the modelling of neuronal cells -see [14], [15], as well as in social sciences [16]. Furthermore, synchronization of networks with dynamic interconnections was recently studied in [17], [18]. In this paper we consider networks under diffusive coupling.

The structural properties of the network are usually described via Graph theory. Generally speaking, a graph may be characterized by a triple $\{\mathcal{V}, \mathcal{E}, L\}$. The first element, $\mathcal{V}$, corresponds to a set of $N$ nodes, $\mathcal{E}$ denotes a set of edges, and $L$ is the so-called Laplacian matrix. This is defined as $L:=D-A$ where $A$ denotes the adjacency matrix, $A=\left[a_{i j}\right]$, whose $(i, j)$ th entry, denoted by $a_{i j}$, specifies the weight of the interconnection between the $i$ th and $j$ th nodes and $D$ corresponds to the degree matrix, which is diagonal and whose $i$ th entry equals to the sum of weights, that is, $\sum_{j=1}^{N} a_{i j}$. If the edges have orientation, the graph is said to be directed; otherwise, it is undirected. See, for instance, [19], [10] for further details. In this paper we consider networks described by directed graphs.

The nodes' dynamics are more commonly represented by continuous-time nonlinear models

$$
\begin{aligned}
& \dot{\boldsymbol{x}}_{i}=f_{i}\left(\boldsymbol{x}_{i}\right)+g_{i}\left(\boldsymbol{x}_{i}, \boldsymbol{u}_{i}\right), \\
& \boldsymbol{y}_{i}=h_{i}\left(\boldsymbol{x}_{i}\right), \quad i \in \mathcal{I}:=\{1, \ldots, N\}
\end{aligned}
$$

where $\boldsymbol{x}_{i} \in \mathbb{R}^{n}, \boldsymbol{u}_{i} \in \mathbb{R}^{m}$ and $\boldsymbol{y}_{i} \in \mathbb{R}^{m}$ denote the state, the input, and the output of the $i$ th unit, respectively. However, analysis and control of networks of systems with hybrid dynamics have become a popular area of research during the last decade -see e.g., [20], [21]. In this paper we consider nonlinear systems in normal affine form, as for instance in [22], [23].

Furthermore, depending on whether the nodes are identical or not the network is respectively called homogeneous or heterogeneous. In the former case, synchronization is often described in terms of the asymptotically identical evolution of the units. For instance, in the simplest consensus paradigm we have $\boldsymbol{x}_{i} \rightarrow \boldsymbol{x}_{j} \rightarrow c$ where $c$ corresponds to the average of the initial conditions -see [10]. More complex asymptotic behaviors are also plausible. For example, the trajectories of network-interconnected oscillators tend to a closed orbit whereas in the problem of formation tracking control the units are required to follow a reference trajectory, that is, $\boldsymbol{x}_{i} \rightarrow \boldsymbol{x}_{j} \rightarrow \boldsymbol{x}^{*}(t)$. Whether a set-point equilibrium or a 
reference trajectory, if we define consensus as the property of all networked systems reaching a state $\boldsymbol{x}_{s} \in \mathbb{R}^{n}$, it is natural to recast this problem as one of asymptotic stability, or stabilization for that effect, of a synchronization manifold

$\mathcal{S}_{x}=\left\{\boldsymbol{x} \in \mathbb{R}^{n N}: \boldsymbol{x}_{1}-\boldsymbol{x}_{s}=\boldsymbol{x}_{2}-\boldsymbol{x}_{s}=\cdots=\boldsymbol{x}_{N}-\boldsymbol{x}_{s}=0\right\}$

-cf. [23], [22]. Moreover, by defining the synchronization errors as $\boldsymbol{e}:=\left[\boldsymbol{e}_{1}^{\top} \cdots \boldsymbol{e}_{N}^{\top}\right]^{\top}$, where $\boldsymbol{e}_{i}=\boldsymbol{x}_{i}-\boldsymbol{x}_{s}$, it follows that $\boldsymbol{x} \in \mathcal{S}_{x}$ if and only if $\boldsymbol{e}=0$. Hence, the general synchronization problem is recasted in the study of stability of the synchronization manifold.

For networks of heterogeneous or homogeneous systems, indistinctly, the definition of $\boldsymbol{x}_{s}$ is instrumental since its dynamics determines the network's mean-field behavior. For instance, in the case of the "classical" consensus paradigm for simple integrators, the network dynamics is completely determined by the Laplacian, hence, $\dot{\boldsymbol{x}}=-L \boldsymbol{x}$ and $\boldsymbol{x}_{s}$ is constant. Moreover, in the particular cases that the graph is undirected or balanced, as in [9], [6], [24], $\boldsymbol{x}_{s}$ corresponds to the average of the systems' states, whereas if the graph is directed and has a spanning tree, $\boldsymbol{x}_{s}$ corresponds to a linear combination of the states $\boldsymbol{x}_{i}$, the weighted average:

$$
\boldsymbol{x}_{s}=\sum_{i=1}^{N} \nu_{i} \boldsymbol{x}_{i}, \quad \sum_{i=1}^{N} \nu_{i}=1
$$

—see, e.g., [25], [26], [27].

In general, however, there is no guarantee for the existence of a constant vector that attracts the systems' solutions, but $\boldsymbol{x}_{s}$ may have its own dynamics. In the case of homogeneous networks one may expect $\boldsymbol{x}_{s}$ to have a similar behavior to that of a single individual. The paradigm is much more complex in the case of heterogeneous networks due to the fact that the synchronization manifold does not necessarily exist and the dynamics of $\boldsymbol{x}_{s}$ may be highly complex and differ from the one of any of the individual agents. For instance, it may possess one equilibrium, multiple equilibria, a limit cycle, a strange attractor, etc. Thus, the trajectories may tend to an attractor that is proper to the collective-behavior dynamics.

These considerations not only motivate a broad definition of the state $\boldsymbol{x}_{s}$ but they also show that, in general, analyzing the stability of the synchronization manifold $\mathcal{S}_{x}$ is insufficient to characterize completely the network behavior.

The conceptual contribution of this paper is to recognize that the collective behavior in network-interconnected systems is dichotomic: on one hand, it consists in the dynamics of a "weighted averaged" motion generated by the so-called meanfield node and, on the other, it is described by the dynamics of each individual unit of the network relative to the dynamics of the mean-field's.

Based on the observation of this duality, we present an original analysis framework constructed upon the concept of emergent dynamics. This pertains to an "average" of the units" drifts and it is distinguished from the mean-field dynamics in that the latter corresponds to a dynamic system whose solutions correspond to an average of the units' trajectories. As we show, the emergent dynamics is intrinsic to the network: it is determined by the connection graph but it is independent of the interconnection strength. From a dynamical systems viewpoint, it corresponds to the dynamics of the mean-field unit, restricted to the synchronization manifold.

Then, we say that the networked systems reach dynamic consensus if, due to their interconnection, all units' motions converge to the one generated by the emergent dynamics. Dynamic consensus covers, for instance, set-point consensus as it has been thoroughly studied in the past years -[10], as well as controlled synchronization of virtual ecosystems [26], [27]. In [10] the emerging "motion" corresponds to an equilibrium point and the emergent dynamics is null. In [26], [27] synchronization is achieved as an agreement on a desired pattern that appears in view of the presence of a common virtual exosystem (the emergent dynamics). The concept of dynamic consensus also generalizes that of consensus with respect to a (compact) set or trajectory $t \mapsto \boldsymbol{x}$ therefore, it includes synchronization problems in the framework of convergent systems.

In accordance with the dichotomy of the collective behavior previously described, we broach the analysis problem by decomposing it in the study of two properties, in terms of stability of sets. On one hand, we assume that the emergent dynamics possesses an attractor $\mathcal{A}$ and we study the motion of the mean-field unit with respect to this attractor. On the other hand, we broach the synchronization problem in terms of the stability of a synchronization manifold which, as in the case of (2), may be defined in terms of the state synchronization errors or, more generally, it may be defined in terms of output synchronization errors.

From a technical view point, our framework establishes a qualitative and formal description of practical synchronization of heterogeneous systems; we establish general results on practical asymptotic stability of the synchronization manifold. More precisely, we show that the synchronization error, which is directly related to the degree of heterogeneity, may be diminished by increasing the interconnection gain. Thus, dynamic consensus may be expected in homogeneous networks whereas in a heterogeneous setting, practical synchronization is achievable in general. Furthermore, we establish conditions on practical stability of the attractor of the emergent dynamics

This paper is the outgrowth of [28], [29], [30]. See also [31] where some preliminary but general results are established for heterogeneous networks described by undirected graphs and the case-study of Andronov-Hopf oscillators is addressed.

The rest of the paper is organized as follows. In Section II we present the problem formulation. In Section III we describe the fundamentals of our analysis framework. In Section IV we derive an error-dynamics model that is suitable for the study of network collective behavior. In Section V we present our main technical statements. In Section VI we illustrate our theoretical findings with a case-study that concerns chaotic oscillators. Finally, we conclude with some remarks in Section VII. 


\section{MODEL AND PROBLEM FORMULATION}

\section{A. System model}

We consider a network composed of $N$ heterogeneous diffusively coupled nonlinear dynamical systems in normal form:

$$
\begin{aligned}
& \dot{\boldsymbol{y}}_{i}=f_{i}^{y}\left(\boldsymbol{y}_{i}, \boldsymbol{z}_{i}\right)+\boldsymbol{u}_{i} \\
& \dot{\boldsymbol{z}}_{i}=f_{i}^{z}\left(\boldsymbol{y}_{i}, \boldsymbol{z}_{i}\right) .
\end{aligned}
$$

As it may be clear from the notation, each unit possesses one input $\boldsymbol{u}_{i}$, and one output $\boldsymbol{y}_{i}$ of the same dimension, i.e., $\boldsymbol{u}_{i}$, $\boldsymbol{y}_{i} \in \mathbb{R}^{m}$. The state $\boldsymbol{z}_{i}$ corresponds to that of the $i$ th agent's zero-dynamics -see [32]. The functions $f_{i}^{y}: \mathbb{R}^{m} \times \mathbb{R}^{n-m} \rightarrow$ $\mathbb{R}^{m}, f_{i}^{z}: \mathbb{R}^{m} \times \mathbb{R}^{n-m} \rightarrow \mathbb{R}^{n-m}$ are assumed to be locally Lipschitz. We assume that the systems are heterogeneous, that is, the functions $f_{i}$ are, in general, different but of the same dimension (we describe the network model in detail farther below).

We also assume that the units possess certain physical properties reminiscent of energy dissipation and propagation. Notably, one of our main hypotheses is that the solutions are ultimately bounded; we recall the definition below.

Definition 1 (Ultimate boundedness) The solutions of the system $\dot{\boldsymbol{x}}=f(\boldsymbol{x}),\left(t, \boldsymbol{x}_{\circ}\right) \mapsto \boldsymbol{x}$, are said to be ultimately bounded if there exist positive constants $\Delta_{\circ}$ and $B_{x}$ such that for every $\Delta \in\left(0, \Delta_{\circ}\right)$, there exists a positive constant $T(\Delta)$ such that, for all $\boldsymbol{x}_{\circ} \in \mathcal{B}_{\Delta}=\left\{\boldsymbol{x} \in \mathbb{R}^{n}:|\boldsymbol{x}| \leq \Delta\right\}$ they satisfy

$$
\left|\boldsymbol{x}\left(t, \boldsymbol{x}_{\circ}\right)\right| \leq B_{x} \quad \text { for all } t \geq T .
$$

If this bound holds with $\Delta_{\circ}=\infty$ then the solutions are globally ultimately bounded.

The importance of this property is that the ultimate bound does not depend on the size of the initial conditions. Moreover, ultimate boundedness is a reasonable assumption for many physical systems. In general, it may be established under Lyapunov-like conditions -see [33] and Section V. In the context of networked systems, ultimate boundedness may be established under the condition that the units are semi-passive -cf. [22], [34], [35], [36].

We also make a standing hypothesis concerning the zerodynamics that is reminiscent of incremental stability:

Assumption 1 There exist compact sets $\mathbb{B}_{z} \subset \mathbb{R}^{n-m}, \mathbb{B}_{y} \subset$ $\mathbb{R}^{m}, N$ continuously differentiable positive definite functions $V_{\circ k}: \mathbb{B}_{z} \rightarrow \mathbb{R}_{+}$with $k \leq N$, class $\mathcal{K}_{\infty}$ functions $\gamma_{1 k}, \gamma_{2 k}$, and constants $\bar{\alpha}_{k}, \beta_{k}>0$ such that, for all $\boldsymbol{z}, \boldsymbol{z}^{\prime} \in \mathbb{B}_{z}$ and $\boldsymbol{y} \in \mathbb{B}_{y}$,

$$
\begin{gathered}
\gamma_{1 k}(|\boldsymbol{z}|) \leq V_{\circ k}(\boldsymbol{z}) \leq \gamma_{2 k}(|\boldsymbol{z}|) \\
\nabla V_{\circ k}\left(\boldsymbol{z}-\boldsymbol{z}^{\prime}\right)\left[f_{k}^{z}(\boldsymbol{y}, \boldsymbol{z})-f_{k}^{z}\left(\boldsymbol{y}, \boldsymbol{z}^{\prime}\right)\right] \leq-\bar{\alpha}_{k}\left|\boldsymbol{z}-\boldsymbol{z}^{\prime}\right|^{2}+\beta_{k}
\end{gathered}
$$

where $\nabla V_{\circ k}:=\frac{\partial V_{\circ k}}{\partial \boldsymbol{z}}$.

Note that Assumption 1 may be viewed as a condition of incremental stability of the zero-dynamics in a practical sense. Indeed, when $\beta_{k}=0$ we recover the characterization provided in [37].

\section{B. Network model}

We assume that the network units are connected via diffusive coupling, i.e., for the $i$ th unit the coupling is given by

$$
\boldsymbol{u}_{i}=-\sigma \sum_{j=1}^{N} a_{i j}\left(\boldsymbol{y}_{i}-\boldsymbol{y}_{j}\right), \quad a_{i j} \geq 0
$$

where the scalar $\sigma$ corresponds to the coupling gain between the units. The weights of the interconnections amongst the nodes are used to define the adjacency matrix, $A=\left[a_{i j}\right]_{i, j \in \mathcal{I}}$, which, in turn, is utilized to construct the corresponding Laplacian matrix,

$$
L=\left[\begin{array}{cccc}
\sum_{i=2}^{N} a_{1 i} & -a_{12} & \ldots & -a_{1 N} \\
-a_{21} & \sum_{i=1, i \neq 2}^{N} a_{2 i} & \ldots & -a_{2 N} \\
\vdots & \vdots & \ddots & \vdots \\
-a_{N 1} & -a_{N 2} & \ldots & \sum_{i=1}^{N-1} a_{N i} .
\end{array}\right]
$$

We stress that in this paper we assume that the graph is directed and strongly connected.

Next, we introduce a compact notation that is convenient for our analysis purposes. We introduce the vectors of outputs, inputs and states, respectively, by

$$
\begin{gathered}
\boldsymbol{y}=\left[\begin{array}{c}
\boldsymbol{y}_{1} \\
\vdots \\
\boldsymbol{y}_{N}
\end{array}\right] \in \mathbb{R}^{m N}, \quad \boldsymbol{u}=\left[\begin{array}{c}
\boldsymbol{u}_{1} \\
\vdots \\
\boldsymbol{u}_{N}
\end{array}\right] \in \mathbb{R}^{m N}, \\
\boldsymbol{x}=\left[\begin{array}{c}
\boldsymbol{x}_{1} \\
\vdots \\
\boldsymbol{x}_{N}
\end{array}\right] \in \mathbb{R}^{n N}, \quad \boldsymbol{x}_{i}=\left[\begin{array}{c}
\boldsymbol{y}_{i} \\
\boldsymbol{z}_{i}
\end{array}\right] \in \mathbb{R}^{n}
\end{gathered}
$$

and the function $F: \mathbb{R}^{n N} \rightarrow \mathbb{R}^{n N}$ is defined as

$$
F(\boldsymbol{x})=\left[\begin{array}{c}
F_{1}\left(\boldsymbol{x}_{1}\right) \\
\vdots \\
F_{N}\left(\boldsymbol{x}_{N}\right)
\end{array}\right], \quad F_{i}\left(\boldsymbol{x}_{i}\right)=\left[\begin{array}{c}
f_{i}^{y}\left(\boldsymbol{y}_{i}, \boldsymbol{z}_{i}\right) \\
f_{i}^{z}\left(\boldsymbol{y}_{i}, \boldsymbol{z}_{i}\right)
\end{array}\right]_{i \in \mathcal{I}} .
$$

With this notation, the diffusive coupling inputs $\boldsymbol{u}_{i}$, defined in (8), can be re-written in the compact form

$$
\boldsymbol{u}=-\sigma\left[L \otimes I_{m}\right] \boldsymbol{y},
$$

where the symbol $\otimes$ stands for the right Kronecker product ${ }^{2}$. Then, the network dynamics becomes

$$
\begin{aligned}
\dot{\boldsymbol{x}} & =F(\boldsymbol{x})-\sigma\left[L \otimes E_{m}\right] \boldsymbol{y} \\
\boldsymbol{y} & =\left[I_{N} \otimes E_{m}^{\top}\right] \boldsymbol{x},
\end{aligned}
$$

where $E_{m}^{\top}=\left[I_{m}, 0_{m \times(n-m)}\right]$.

\section{Collective BehaVior IN Heterogeneous NETWORKS}

In this section we describe in detail the basis to our qualitative analysis framework for the study of the solutions of the networked systems (11). As it is discussed in the Introduction, our approach is based on the dichotomic nature of

\footnotetext{
${ }^{2}$ Recall that, given $A \in \mathbb{R}^{m \times \ell}$ and $B \in \mathbb{R}^{n \times k}, A \otimes B:=\left[a_{i j} B\right] \in$ $\mathbb{R}^{m n \times \ell k}$.
} 


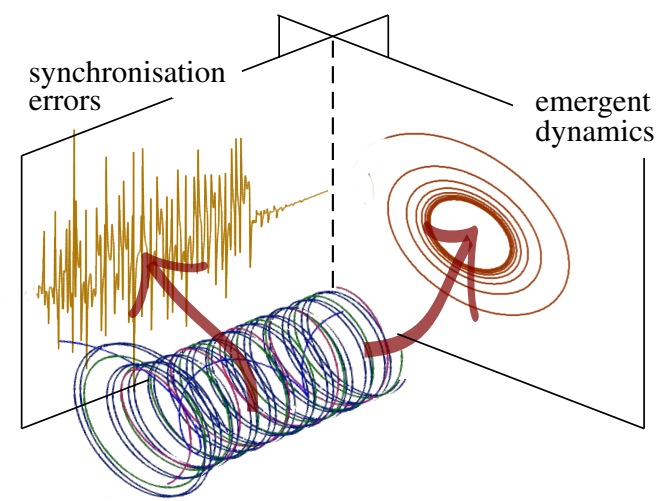

Fig. 1. Dichotomy of dynamic consensus in heterogeneous networks

the network's collective behavior. In accordance, the system's state space is decomposed in two orthogonal subspaces, one on which is projected the behavior of the mean-field state $\boldsymbol{x}_{s}$ and one in which lay the synchronization errors, $e$. See Figure 1 for a schematic illustration.

We show that the states $\boldsymbol{x}_{s}$ and $\boldsymbol{e}$ are intrinsic to the network and not the product of an artifice with purely theoretical motivations. Thus, the study of the motion $t \mapsto\left(\boldsymbol{x}_{s}, \boldsymbol{e}\right)$, projected into the state space of $\left(\boldsymbol{x}_{s}, \boldsymbol{e}\right)$, which is of higher dimension than that of the original dynamics, (11), is instrumental to completely assess the synchronization and the collective behavior properties.

\section{A. Network characterization in new coordinates}

We start by unfolding a natural definition of the mean-field coordinates $\boldsymbol{x}_{s}$ and the synchronization errors $\boldsymbol{e}$, in a general setting.

By construction, all row sums of $L$ are equal to zero -see Eq. (9) hence, it has at least one eigenvalue equal to zero (say, $\lambda_{1}$ ) while the others have non-negative real parts. Moreover, assuming that the network graph is strongly connected, it follows that $\lambda_{1}$ has algebraic multiplicity equal to one and the others have positive real parts, i.e., $0=\Re e\left\{\lambda_{1}\right\}<\Re e\left\{\lambda_{2}\right\} \leq$ $\cdots \leq \Re e\left\{\lambda_{N}\right\}$. Furthermore, if the graph is directed and has a spanning tree the right and left eigenvectors corresponding to $\lambda_{1}$, denoted $v_{r 1} \in \mathbb{R}^{N}$ and $v_{\ell 1} \in \mathbb{R}^{N}$, are given by

$$
v_{r 1}=\mathbf{1}_{N}:=\left[\begin{array}{c}
1 \\
\vdots \\
1
\end{array}\right], \quad v_{\ell 1}=\left[\begin{array}{c}
\nu_{1} \\
\vdots \\
\nu_{N}
\end{array}\right]
$$

where $\nu_{i}>0$ for all $i \in \mathcal{I}$-see [38].

It is convenient to remark that, similarly to (3), the weighted average is defined using the vector $\vartheta_{\ell 1}$, that is, $\boldsymbol{x}_{s}$ is a projection of $\boldsymbol{x}$ on the subspace generated by $\vartheta_{\ell 1}$. Moreover, for the Laplacian $L$ there exists a Jacobian decomposition of the form $L=U \Lambda U^{-1}$ where $U \in \mathbb{C}^{N \times N}$ is nonsingular and $\Lambda \in \mathbb{C}^{N \times N}$ is a block-diagonal Jordan matrix

$$
\Lambda:=\left[\begin{array}{ccc}
\Lambda_{1} & & 0 \\
& \ddots & \\
0 & & \Lambda_{m}
\end{array}\right]
$$

where, for a network with a spanning tree, $\Lambda_{1}$ is scalar and equals to zero and $\Lambda_{i}$ are Jordan blocks of appropriate dimensions. Furthermore, the matrix $U$ is composed of generalized right-eigenvectors of $L$ among which the first is $v_{r 1}=\mathbf{1}_{N}$ hence, for further development, we decompose $U$ as

$$
U=\left[\begin{array}{ll}
\mathbf{1}_{N} & U_{1}
\end{array}\right]
$$

where $U_{1} \in \mathbb{C}^{N \times N-1}$. In this case, the first row of $U^{-1}$ corresponds to the first left eigen-vector of $L, v_{\ell 1}$. Therefore, we may decompose $U^{-1}$ as

$$
U^{-1}=\left[\begin{array}{l}
v_{\ell 1}^{\top} \\
U_{1}^{\dagger}
\end{array}\right]
$$

and, necessarily,

$$
v_{\ell 1}^{\top} U_{1}=0, \quad U_{1}^{\dagger} U_{1}=I_{N-1} .
$$

Based on the latter observations we introduce the coordinate transformation

$$
\overline{\boldsymbol{x}}:=\mathcal{U}^{-1} \boldsymbol{x},
$$

where the block diagonal matrix $\mathcal{U} \in \mathbb{C}^{n N \times n N}$ is defined as

$$
\mathcal{U}=U \otimes I_{n} .
$$

Then, we use (15) and (16) to partition the new coordinates $\bar{x}$, i.e.,

$$
\overline{\boldsymbol{x}}=:\left[\begin{array}{c}
\overline{\boldsymbol{x}}_{1} \\
\overline{\boldsymbol{x}}_{2}
\end{array}\right]:=\left[\begin{array}{c}
v_{\ell 1}^{\top} \otimes I_{n} \\
U_{1}^{\dagger} \otimes I_{n}
\end{array}\right] \boldsymbol{x} .
$$

The coordinates $\overline{\boldsymbol{x}}_{1}$ and $\overline{\boldsymbol{x}}_{2}$ thus obtained are equivalent to the weighted average $\boldsymbol{x}_{s}$ and the synchronization errors $\boldsymbol{e}$, respectively. Indeed, observing that the state of the mean-field unit, defined in (3), may be re-written in the compact form

$$
\boldsymbol{x}_{s}=\left[v_{\ell 1}^{\top} \otimes I_{n}\right] \boldsymbol{x},
$$

we see that $\overline{\boldsymbol{x}}_{1}=\boldsymbol{x}_{s}$. Moreover, $\overline{\boldsymbol{x}}_{2}=0$ if and only if $\boldsymbol{e}=0$. To see the latter, let $\mathcal{U}_{1}:=U_{1} \otimes I_{n}$ and $\mathcal{U}_{1}^{\dagger}:=U_{1}^{\dagger} \otimes I_{n}$. Then, using the expression

$$
(A \otimes B)(C \otimes D)=A C \otimes B D,
$$

we obtain

$$
\mathcal{U}_{1} \mathcal{U}_{1}^{\dagger}=\left[U_{1} U_{1}^{\dagger}\right] \otimes I_{n}
$$

and, observing that

$$
U_{1} U_{1}^{\dagger}=I_{N}-\mathbf{1}_{N} v_{\ell 1}^{\top},
$$

we get

$$
\mathcal{U}_{1} \mathcal{U}_{1}^{\dagger}=\left[I_{N}-\mathbf{1}_{N} v_{\ell 1}^{\top}\right] \otimes I_{n} .
$$

So, premultiplying $\bar{x}_{2}=\mathcal{U}_{1}^{\dagger} \boldsymbol{x}$ by $\mathcal{U}_{1}$ and using (20), we obtain

$$
\begin{aligned}
\mathcal{U}_{1} \overline{\boldsymbol{x}}_{2} & =\left[\left(I_{N}-\mathbf{1}_{N} v_{\ell 1}^{\top}\right) \otimes I_{n}\right] \boldsymbol{x} \\
& =\boldsymbol{x}-\left[\left(\mathbf{1}_{N} v_{\ell 1}^{\top}\right) \otimes I_{n}\right] \boldsymbol{x}
\end{aligned}
$$

which, in view of (19), is equivalent to

$$
\begin{aligned}
\mathcal{U}_{1} \overline{\boldsymbol{x}}_{2} & =\boldsymbol{x}-\left[\mathbf{1}_{N} \otimes I_{n}\right]\left[v_{\ell 1}^{\top} \otimes I_{n}\right] \boldsymbol{x} \\
& =\boldsymbol{x}-\left[\mathbf{1}_{N} \otimes I_{n}\right] \boldsymbol{x}_{s}=\boldsymbol{e} .
\end{aligned}
$$


Hence, since $\mathcal{U}_{1}$ has column rank equal to $(N-1) n$, which corresponds to the dimension of $\overline{\boldsymbol{x}}_{2}$, we see that $\overline{\boldsymbol{x}}_{2}$ is equal to zero if and only if so is $e$.

We finish this section with a technical statement which applies, in particular, to the analysis of synchronization of networked integrators interconnected through a directed graph. Even though similar results may be found in the literature (see e.g. [39], [26], [27]) the specific construction of the Lyapunov function given in Lemma 1 is used in our main result. For the sake of completeness, the proof is provided in Appendix A.

Lemma 1 Consider the system $\dot{\boldsymbol{x}}=-L \boldsymbol{x}$, where $\boldsymbol{x} \in \mathbb{R}^{N}$ and the matrix $L \in \mathbb{R}^{N \times N}$ has exactly one zero eigenvalue and all others have positive real parts. Let $v_{\ell 1}, v_{r 1} \in \mathbb{R}^{N}$ denote, respectively, left and right eigen-vectors ${ }^{3}$ corresponding to the zero eigenvalue.

Let, without loss of generality, $v_{\ell 1}^{\top} v_{r 1}=1$ and define the errors

$$
\boldsymbol{e}:=\boldsymbol{x}-v_{r 1} v_{\ell 1}^{\top} \boldsymbol{x}
$$

Then, the set

$$
\mathcal{S}_{x}:=\left\{\boldsymbol{x} \in \mathbb{R}^{N}: \boldsymbol{e}=0\right\}
$$

is globally exponentially stable. Moreover, there exists a symmetric, positive definite matrix $\Gamma \in \mathbb{R}^{N \times N}$ and a constant $\gamma>0$ such that the function $V: \mathbb{R}^{N} \rightarrow \mathbb{R}_{\geq 0}$, defined as

$$
V(e)=e^{\top} \Gamma e
$$

satisfies

$$
\dot{V}(\boldsymbol{e}) \leq-\gamma|\boldsymbol{e}|^{2} .
$$

Remark 1 The statement of Lemma 1 is not restricted to linear systems in which $L$ has positive off-diagonal entries. However, in the particular case that $L$ corresponds to a directed graph with a spanning tree (hence, such that in addition to the hypothesis of Lemma $1, L$ is row-stochastic and its off-diagonal elements are positive), the synchronization error becomes $\boldsymbol{e}:=\boldsymbol{x}-\mathbf{1}_{N} v_{\ell 1}^{\top} \boldsymbol{x}$ and we recover [39, Lemma 3.1].

\section{B. Dynamic consensus, mean-field and emergent dynamics}

Now that we have defined the mean-field state $\boldsymbol{x}_{s}$ we proceed to derive its dynamics equation. To that end, with the purpose of studying output synchronization, let us partition this variable into the mean-field output, $\boldsymbol{y}_{s} \in \mathbb{R}^{m}$, defined as $\boldsymbol{y}_{s}=E_{m}^{\top} \boldsymbol{x}_{s}$ and the state of the mean-field zero dynamics, $\boldsymbol{z}_{s} \in \mathbb{R}^{n-m}$, that is, $\boldsymbol{x}_{s}=\left[\boldsymbol{y}_{s}^{\top}, \boldsymbol{z}_{s}^{\top}\right]^{\top}$.

Then, by differentiating on both sides of (18) and after a direct computation in which we use (4), (8) and the fact that $v_{\ell 1}^{\top} L=0$, we obtain

$$
\begin{aligned}
\dot{\boldsymbol{y}}_{s} & =\sum_{i=1}^{N} \nu_{i} f_{i}^{y}\left(\boldsymbol{y}_{i}, \boldsymbol{z}_{i}\right), \\
\dot{\boldsymbol{z}}_{s} & =\sum_{i=1}^{N} \nu_{i} f_{i}^{z}\left(\boldsymbol{y}_{i}, \boldsymbol{z}_{i}\right)
\end{aligned}
$$

\footnotetext{
${ }^{3}$ Since $L$ is real one can always find real eigenvectors — see [40, Section 3.2].
}

where the constants $\nu_{i}$ are defined in (12). Next, for these equations to be meaningful, we rewrite them in terms of the state $\boldsymbol{x}_{s}$. To that end, we introduce the functions $f_{s}^{y}: \mathbb{R}^{m} \times$ $\mathbb{R}^{n-m} \rightarrow \mathbb{R}^{m}, f_{s}^{z}: \mathbb{R}^{m} \times \mathbb{R}^{n-m} \rightarrow \mathbb{R}^{n-m}$ and we define them as

$$
\begin{aligned}
f_{s}^{y}\left(\boldsymbol{y}_{s}, \boldsymbol{z}_{s}\right) & :=\sum_{i=1}^{N} \nu_{i} f_{i}^{y}\left(\boldsymbol{y}_{s}, \boldsymbol{z}_{s}\right), \\
f_{s}^{z}\left(\boldsymbol{y}_{s}, \boldsymbol{z}_{s}\right) & :=\sum_{i=1}^{N} \nu_{i} f_{i}^{z}\left(\boldsymbol{y}_{s}, \boldsymbol{z}_{s}\right)
\end{aligned}
$$

so, adding and subtracting $f_{s}^{y}\left(\boldsymbol{y}_{s}, \boldsymbol{z}_{s}\right)$ to (24a) and $f_{s}^{z}\left(\boldsymbol{y}_{s}, \boldsymbol{z}_{s}\right)$ to $(24 b)$, we see that (24) is equivalent to

$$
\begin{aligned}
& \dot{\boldsymbol{y}}_{s}=f_{s}^{y}\left(\boldsymbol{y}_{s}, \boldsymbol{z}_{s}\right)+\sum_{i=1}^{N} \nu_{i}\left[f_{i}^{y}\left(\boldsymbol{y}_{i}, \boldsymbol{z}_{i}\right)-f_{i}^{y}\left(\boldsymbol{y}_{s}, \boldsymbol{z}_{s}\right)\right], \\
& \dot{\boldsymbol{z}}_{s}=f_{s}^{z}\left(\boldsymbol{y}_{s}, \boldsymbol{z}_{s}\right)+\sum_{i=1}^{N} \nu_{i}\left[f_{i}^{z}\left(\boldsymbol{y}_{i}, \boldsymbol{z}_{i}\right)-f_{i}^{z}\left(\boldsymbol{y}_{s}, \boldsymbol{z}_{s}\right)\right] .
\end{aligned}
$$

The latter equations may be regarded as composed of the nominal subsystem

$$
\begin{aligned}
& \dot{\boldsymbol{y}}_{s}=f_{s}^{y}\left(\boldsymbol{y}_{s}, \boldsymbol{z}_{s}\right) \\
& \dot{\boldsymbol{z}}_{s}=f_{s}^{z}\left(\boldsymbol{y}_{s}, \boldsymbol{z}_{s}\right)
\end{aligned}
$$

which is affected by the perturbation terms $\left[f_{i}^{y}\left(\boldsymbol{y}_{i}, \boldsymbol{z}_{i}\right)-\right.$ $\left.f_{i}^{y}\left(\boldsymbol{y}_{s}, \boldsymbol{z}_{s}\right)\right]$ and $\left[f_{i}^{z}\left(\boldsymbol{y}_{i}, \boldsymbol{z}_{i}\right)-f_{i}^{z}\left(\boldsymbol{y}_{s}, \boldsymbol{z}_{s}\right)\right]$. With the purpose of underlining the role of these two components, notice that the perturbations vanish if and only if $\left(\boldsymbol{y}_{i}, \boldsymbol{z}_{i}\right) \rightarrow\left(\boldsymbol{y}_{s}, \boldsymbol{z}_{s}\right)$.

On the other hand, in compact form, the nominal subsystem (27) may be expressed as

$$
\dot{\boldsymbol{x}}_{e}=f_{s}\left(\boldsymbol{x}_{e}\right) \quad \boldsymbol{x}_{e}=\left[\boldsymbol{y}_{e}^{\top} \boldsymbol{z}_{e}^{\top}\right]^{\top}, \quad f_{s}:=\left[f_{s}^{y^{\top}} f_{s}^{z^{\top}}\right]^{\top}
$$

in which we denote the state by $\boldsymbol{x}_{e}$ to emphasize the distinction between (28) and (26). We refer to the former as the emergent dynamics and to the latter as mean-field dynamics. In other words, the emergent dynamics determines the behavior of the mean-field solutions, restricted to the synchronization manifold $\{\boldsymbol{e}=0\}$.

Exhibiting the existence of the emergent dynamics naturally leads to a significant generalization of the consensus paradigm, that of dynamic consensus. We shall say that this property is achieved by the systems interconnected over a network if their motions converge to one generated by (28). Back to our comparison with the classical (set-point) consensus paradigm, in which all systems' trajectories converge to a common equilibrium point, note that in this case we have $f_{s} \equiv 0$ and $\boldsymbol{x}_{e}$ is a constant that linearly depends on the initial state values. In the example of formation tracking control, Equation (28) can be seen as the reference dynamics for the formation.

\section{NETWORK DYNAMICS}

In accordance with the dichotomic nature of dynamic consensus -see Figure 1, our study relies on the qualitative stability analysis of two dynamical subsystems: the first corresponds to the mean-field system with state $\boldsymbol{x}_{s}$ and the second defines the evolution of the synchronization errors $e$. From a systems viewpoint, this representation corresponds to that of two feedback interconnected blocks -see Figure 2. 


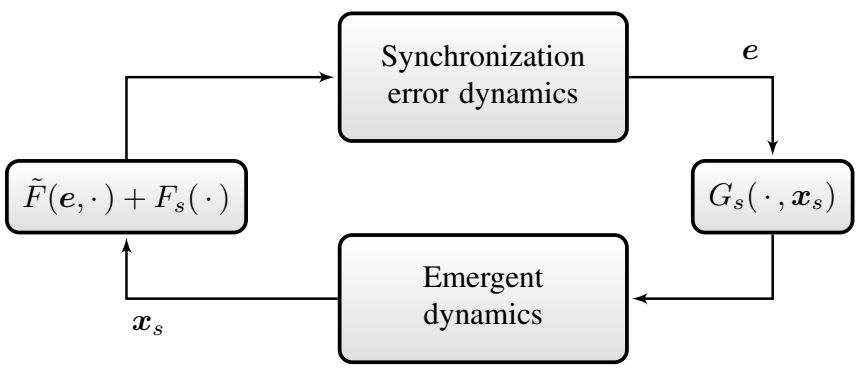

Fig. 2. Interaction between synchronization and the collective dynamics

\section{A. Dynamics of the mean-field subsystem}

Using the network dynamics equations (11a), as well as (18), we obtain

$$
\dot{\boldsymbol{x}}_{s}=\left[v_{\ell 1}^{\top} \otimes I_{n}\right] F(\boldsymbol{x})-\sigma\left[v_{\ell 1}^{\top} \otimes I_{n}\right]\left[L \otimes E_{m}\right] \boldsymbol{y} .
$$

Now, using the property of the Kronecker product that is given in (19), and in view of the identity $v_{\ell 1}^{\top} L=0$, we obtain

$$
\left[v_{\ell 1}^{\top} \otimes I_{n}\right]\left[L \otimes E_{m}\right]=\left[v_{\ell 1}^{\top} L\right] \otimes\left[I_{n} E_{m}\right]=0 .
$$

Therefore, (29) becomes $\dot{\boldsymbol{x}}_{s}=\left[v_{\ell 1}^{\top} \otimes I_{n}\right] F(\boldsymbol{x})$. This reveals the important fact that the mean-field dynamics is independent of the interconnections gain $\sigma$, even though the solutions $\boldsymbol{x}_{s}(t)$ are, certainly, affected by the synchronization errors hence, by the coupling strength.

Next, we use (10) and we define

$$
f_{s}\left(\boldsymbol{x}_{s}\right):=\sum_{i=1}^{N} \nu_{i} F_{i}\left(\boldsymbol{x}_{s}\right)
$$

to obtain

$$
\dot{\boldsymbol{x}}_{s}=f_{s}\left(\boldsymbol{x}_{s}\right)+\sum_{i=1}^{N} \nu_{i}\left[F_{i}\left(\boldsymbol{x}_{i}\right)-F_{i}\left(\boldsymbol{x}_{s}\right)\right] .
$$

Therefore, defining

$$
G_{s}\left(\boldsymbol{e}, \boldsymbol{x}_{s}\right):=\sum_{i=1}^{N} \nu_{i}\left[F_{i}\left(\boldsymbol{e}_{i}+\boldsymbol{x}_{s}\right)-F_{i}\left(\boldsymbol{x}_{s}\right)\right],
$$

the mean-field dynamics equation takes the compact form

$$
\dot{\boldsymbol{x}}_{s}=f_{s}\left(\boldsymbol{x}_{s}\right)+G_{s}\left(\boldsymbol{e}, \boldsymbol{x}_{s}\right) \text {. }
$$

Furthermore, since the functions $F_{i}$, with $i \in \mathcal{I}$, are locally Lipschitz so is the function $G_{s}$ and, moreover, there exists a continuous, positive, non-decreasing function $k: \mathbb{R}_{+} \times \mathbb{R}_{+} \rightarrow$ $\mathbb{R}_{+}$, such that

$$
\left|G_{s}\left(\boldsymbol{e}, \boldsymbol{x}_{s}\right)\right| \leq k\left(|\boldsymbol{e}|,\left|\boldsymbol{x}_{s}\right|\right)|\boldsymbol{e}|
$$

\section{B. Dynamics of the synchronization errors}

To study the effect of the synchronization errors, $e$, on the emergent dynamics, we start by introducing the vectors

$$
\begin{aligned}
& F_{s}\left(\boldsymbol{x}_{s}\right):= {\left[\begin{array}{ccc}
F_{1}\left(\boldsymbol{x}_{s}\right)^{\top} & \cdots & F_{N}\left(\boldsymbol{x}_{s}\right)^{\top}
\end{array}\right]^{\top}, } \\
& \tilde{F}\left(\boldsymbol{e}, \boldsymbol{x}_{s}\right):=\left[\begin{array}{c}
F_{1}\left(\boldsymbol{x}_{1}\right)-F_{1}\left(\boldsymbol{x}_{s}\right) \\
\vdots \\
F_{N}\left(\boldsymbol{x}_{N}\right)-F_{N}\left(\boldsymbol{x}_{s}\right)
\end{array}\right]
\end{aligned}
$$

$$
=\left[\begin{array}{c}
F_{1}\left(\boldsymbol{e}_{1}+\boldsymbol{x}_{s}\right)-F_{1}\left(\boldsymbol{x}_{s}\right) \\
\vdots \\
F_{N}\left(\boldsymbol{e}_{N}+\boldsymbol{x}_{s}\right)-F_{N}\left(\boldsymbol{x}_{s}\right)
\end{array}\right] .
$$

That is, $\tilde{F}\left(\boldsymbol{e}, \boldsymbol{x}_{s}\right)=F(\boldsymbol{x})-F_{s}\left(\boldsymbol{x}_{s}\right)$ and $F_{s}\left(\boldsymbol{x}_{s}\right)=F\left(\mathbf{1}_{N} \otimes\right.$ $\left.\boldsymbol{x}_{s}\right)$. Then, differentiating on both sides of

$$
\boldsymbol{e}=\boldsymbol{x}-\left(\mathbf{1}_{N} \otimes I_{n}\right) \boldsymbol{x}_{s}
$$

while using (11a) and (33), we obtain

$$
\begin{aligned}
\dot{\boldsymbol{e}}= & -\sigma\left[L \otimes E_{m}\right] \boldsymbol{y}+F(\boldsymbol{x}) \\
& -\left(\mathbf{1}_{N} \otimes I_{n}\right)\left[f_{s}\left(\boldsymbol{x}_{s}\right)+G_{s}\left(\boldsymbol{e}, \boldsymbol{x}_{s}\right)\right] \\
=- & -\sigma\left[L \otimes E_{m}\right] \boldsymbol{y}+\left[F(\boldsymbol{x})-F_{s}\left(\boldsymbol{x}_{s}\right)\right]+F_{s}\left(\boldsymbol{x}_{s}\right) \\
& -\left(\mathbf{1}_{N} \otimes I_{n}\right)\left[f_{s}\left(\boldsymbol{x}_{s}\right)+G_{s}\left(\boldsymbol{e}, \boldsymbol{x}_{s}\right)\right] \\
= & -\sigma\left[L \otimes E_{m}\right] \boldsymbol{y}+\left[F_{s}\left(\boldsymbol{x}_{s}\right)-\left(\mathbf{1}_{N} \otimes I_{n}\right) f_{s}\left(\boldsymbol{x}_{s}\right)\right] \\
& +\left[\tilde{F}\left(\boldsymbol{e}, \boldsymbol{x}_{s}\right)-\left(\mathbf{1}_{N} \otimes I_{n}\right) G_{s}\left(\boldsymbol{e}, \boldsymbol{x}_{s}\right)\right] .
\end{aligned}
$$

Next, let us introduce the output synchronization errors $\boldsymbol{e}_{y i}=\boldsymbol{y}_{i}-\boldsymbol{y}_{s}, \boldsymbol{e}_{y}=\left[\boldsymbol{e}_{y 1}^{\top}, \cdots, \boldsymbol{e}_{y N}^{\top}\right]^{\top}$, which may also be written as

$$
\boldsymbol{e}_{y}=\boldsymbol{y}-\mathbf{1}_{N} \otimes \boldsymbol{y}_{s}
$$

and let us consider the first term and the two groups of bracketed terms on the right-hand side of (38), separately. For the term $\left(L \otimes E_{m}\right) \boldsymbol{y}$ we observe, from (39), that

$$
\left[L \otimes E_{m}\right] \boldsymbol{y}=\left[L \otimes E_{m}\right]\left[\boldsymbol{e}_{y}+\mathbf{1}_{N} \otimes \boldsymbol{y}_{s}\right]
$$

and we use (19) and the fact that $L \mathbf{1}_{N}=0$ to obtain

$$
\left[L \otimes E_{m}\right] \boldsymbol{y}=\left[L \otimes E_{m}\right] \boldsymbol{e}_{y}
$$

Secondly, concerning the first bracket on the right-hand side of (38) we observe that, in view of (31) and (35),

$$
f_{s}\left(\boldsymbol{x}_{s}\right)=\left[v_{\ell 1}^{\top} \otimes I_{n}\right] F_{s}\left(\boldsymbol{x}_{s}\right)
$$

therefore,

$$
\begin{aligned}
F_{s}\left(\boldsymbol{x}_{s}\right)- & {\left[\mathbf{1}_{N} \otimes I_{n}\right] f_{s}\left(\boldsymbol{x}_{s}\right)=} \\
& F_{s}\left(\boldsymbol{x}_{s}\right)-\left[\mathbf{1}_{N} \otimes I_{n}\right]\left[v_{\ell 1}^{\top} \otimes I_{n}\right] F_{s}\left(\boldsymbol{x}_{s}\right) .
\end{aligned}
$$

Then, using (19) we see that

$$
\left[\mathbf{1}_{N} \otimes I_{n}\right]\left[v_{\ell 1}^{\top} \otimes I_{n}\right]=\left[\mathbf{1}_{N} v_{\ell 1}^{\top}\right] \otimes I_{n}
$$

so, introducing the projection matrix,

$$
\Pi:=I_{n N}-\left[\mathbf{1}_{N} v_{\ell 1}^{\top}\right] \otimes I_{n},
$$

we obtain

$$
F_{s}\left(\boldsymbol{x}_{s}\right)-\left[\mathbf{1}_{N} \otimes I_{n}\right] f_{s}\left(\boldsymbol{x}_{s}\right)=\Pi F_{s}\left(\boldsymbol{x}_{s}\right) .
$$

Finally, regarding the term $\tilde{F}\left(\boldsymbol{e}, \boldsymbol{x}_{s}\right)-\left(\mathbf{1}_{N} \otimes I_{n}\right) G_{s}\left(\boldsymbol{e}, \boldsymbol{x}_{s}\right)$ on the right-hand side of (38), we see that, by definition, $G\left(\boldsymbol{e}, \boldsymbol{x}_{s}\right)=\left(v_{\ell 1}^{\top} \otimes I_{n}\right) \tilde{F}\left(\boldsymbol{e}, \boldsymbol{x}_{s}\right)$ hence, from (40), we obtain

$$
\left(\mathbf{1}_{N} \otimes I_{n}\right) G_{s}\left(\boldsymbol{e}, \boldsymbol{x}_{s}\right)=\left[\left[\mathbf{1}_{N} v_{\ell 1}^{\top}\right] \otimes I_{n}\right] \tilde{F}\left(\boldsymbol{e}, \boldsymbol{x}_{s}\right)
$$

and

$$
\begin{aligned}
\tilde{F}\left(\boldsymbol{e}, \boldsymbol{x}_{s}\right) & -\left(\mathbf{1}_{N} \otimes I_{n}\right) G_{s}\left(\boldsymbol{e}, \boldsymbol{x}_{s}\right) \\
= & {\left[I_{n N}-\left[\mathbf{1}_{N} v_{\ell 1}^{\top}\right] \otimes I_{n}\right] \tilde{F}\left(\boldsymbol{e}, \boldsymbol{x}_{s}\right)=\Pi \tilde{F}\left(\boldsymbol{e}, \boldsymbol{x}_{s}\right) . }
\end{aligned}
$$


Using (41) and (42) in (38) we see that the latter may be expressed as

$$
\dot{\boldsymbol{e}}=-\sigma\left[L \otimes E_{m}\right] \boldsymbol{e}_{y}+\Pi\left[\tilde{F}\left(\boldsymbol{e}, \boldsymbol{x}_{s}\right)+F_{s}\left(\boldsymbol{x}_{s}\right)\right] .
$$

The utility of this equation is that it clearly exhibits three terms: a term linear in the output $e_{y}$ which reflects the synchronization effect of diffusive coupling among the nodes, the term $\Pi \tilde{F}\left(\boldsymbol{e}, \boldsymbol{x}_{s}\right)$ which vanishes with the synchronization errors (that is, if $e=0$ ), and the term

$$
\Pi F_{s}\left(\boldsymbol{x}_{s}\right)=\left[\begin{array}{c}
F_{1}\left(\boldsymbol{x}_{s}\right)-f_{s}\left(\boldsymbol{x}_{s}\right) \\
\vdots \\
F_{N}\left(\boldsymbol{x}_{s}\right)-f_{s}\left(\boldsymbol{x}_{s}\right)
\end{array}\right]
$$

which represents the variation between the dynamics of the individual units and of the mean-field unit. This term equals to zero only when the nominal dynamics (that is $f_{i}^{y}$ and $f_{i}^{z}$ in (4)), of all the units are identical, that is, in the case of a homogeneous network.

We finish this section by remarking, for further development, that in view of Lipschitz continuity there exist nondecreasing functions $\rho_{1}: \mathbb{R}_{>0} \times \mathbb{R}_{>0} \rightarrow \mathbb{R}_{>0}$ and $\rho_{2}$ : $\mathbb{R}_{\geq 0} \times \mathbb{R}_{\geq 0} \rightarrow \mathbb{R}_{\geq 0}$ such that the perturbation terms $\Pi \tilde{F}\left(\boldsymbol{e}, \boldsymbol{x}_{s}\right)$ and $\Pi F_{s}\left(\boldsymbol{x}_{s}\right)$ satisfy:

$$
\begin{aligned}
\left|\Pi \tilde{F}\left(\boldsymbol{e}, \boldsymbol{x}_{s}\right)\right| & \leq \rho_{1}\left(|\boldsymbol{e}|,\left|\boldsymbol{x}_{s}\right|\right) \\
\left|\Pi F_{s}\left(\boldsymbol{x}_{s}\right)\right| & \leq \rho_{2}\left(\left|\boldsymbol{x}_{s}\right|\right)
\end{aligned}
$$

for all $^{4} \boldsymbol{x} \in \mathbb{R}^{n N}$.

Therefore, in view of (43), for each $B_{x}$ there exist $C_{1}$ and $C_{2}$ such that, for all $|\boldsymbol{x}| \leq B_{x}$,

$$
\left|\Pi \tilde{F}\left(\boldsymbol{e}, \boldsymbol{x}_{s}\right)\right| \leq C_{1}\left(B_{x}\right), \quad\left|\Pi F_{s}\left(\boldsymbol{x}_{s}\right)\right| \leq C_{2}\left(B_{x}\right) .
$$

\section{NETWORK STABILITY}

For the purpose of analysis, since the mean-field dynamics is regarded as a "perturbed variant" of the emergent dynamics, it appears natural to study the problem of dynamic consensus, recasted in that of robust stability analysis, in a broad sense -see, e.g., [9], [8], [6].

Firstly, in contrast to the more-commonly studied case of state-synchronization, i.e., the stability analysis of the manifold (2), we shall admit that synchronization may be established with respect to the outputs $\boldsymbol{y}_{i}$ hence, we introduce the output synchronization manifold as

$\mathcal{S}_{y}=\left\{\boldsymbol{y} \in \mathbb{R}^{m N}: \boldsymbol{y}_{1}-\boldsymbol{y}_{s}=\boldsymbol{y}_{2}-\boldsymbol{y}_{s}=\cdots=\boldsymbol{y}_{N}-\boldsymbol{y}_{s}=0\right\}$.

Secondly, since in the general case of heterogeneous networks the manifold $\mathcal{S}_{x}$ cannot be stabilised asymptotically, one may only aspire at establishing stability of the output or state synchronization manifolds $\mathcal{S}_{y}$ or $\mathcal{S}_{x}$ in a practical sense. The property defined below covers practical stability, as used in [41], [42], by considering a stability property with respect to sets.

Consider a parameterized system of differential equations

$$
\dot{\boldsymbol{x}}=f(\boldsymbol{x}, \eta),
$$

\footnotetext{
${ }^{4}$ Recall that $\boldsymbol{e}$ and $\boldsymbol{x}_{s}$ are functions of $\boldsymbol{x}$.
}

where $\boldsymbol{x} \in \mathbb{R}^{q}$ and $\eta$ is a scalar parameter such that $\eta \in$ $\left[\eta_{\circ}, \infty\right)$ with $\eta_{\mathrm{o}}>0$. It is assumed that for each $\eta$, the function $f(\cdot, \eta): \mathbb{R}^{q} \rightarrow \mathbb{R}^{q}$ is locally Lipschitz. We recall that for a closed set $\mathcal{A}$ we define the norm $|\boldsymbol{x}|_{\mathcal{A}}:=\inf _{\boldsymbol{\zeta} \in \mathcal{A}}|\boldsymbol{x}-\boldsymbol{\zeta}|$.

Definition 2 For the system (46), we say that the closed set $\mathcal{A} \subset \mathbb{R}^{n}$ is uniformly practically asymptotically stable if there exists a set $\mathcal{D}$ such that $\mathcal{A} \subset \mathcal{D} \subset \mathbb{R}^{n}$ and:

(1) the system is forward complete ${ }^{5}$ for all $\boldsymbol{x}_{\circ} \in \mathcal{D}$;

(2) for any given $\delta>0$ and $R>0$, there exist $\eta^{*} \in\left[\eta_{\circ}, \infty\right)$ such that, for all $\eta \in\left[\eta^{*}, \infty\right)$, there exists a class $\mathcal{K} \mathcal{L}$ function $\beta_{\eta}$ such that, for all $\boldsymbol{x}_{\circ} \in \mathcal{D}$ satisfying $\left|\boldsymbol{x}_{\circ}\right|_{\mathcal{A}} \leq$ $R$, we have

$$
\left|\boldsymbol{x}\left(t, \boldsymbol{x}_{\circ}, \eta\right)\right|_{\mathcal{A}} \leq \delta+\beta_{\eta}\left(\left|\boldsymbol{x}_{\circ}\right|_{\mathcal{A}}, t\right) .
$$

If $\mathcal{D}=\mathbb{R}^{n}$ then the set $\mathcal{A}$ is uniformly globally practically asymptotically stable.

The proofs of our main results, which are presented farther below, rely on the following statement on practical asymptotic stability. Even though we were unable to locate this statement as such in the literature, its proof may be established by mimicking the method of proof of known statements, such as in [43], [33] on ultimate boundedness or, in [44] on stability of sets. However, to make the paper self-contained, we provide a short direct proof in Appendix B.

Proposition 1 Consider the system (46) with $\mathcal{D}=\mathbb{R}^{n}$ and assume that it is forward complete. Assume, further, that there exist a $C^{1}$ function $V: \mathbb{R}^{n} \rightarrow \mathbb{R}_{+}$as well as functions $\alpha_{1}, \alpha_{2}, \alpha_{3} \in \mathcal{K}_{\infty}$ and a constant $c>0$, such that, for all $\boldsymbol{x} \in \mathbb{R}^{n}$,

$$
\begin{gathered}
\alpha_{1}\left(|\boldsymbol{x}|_{\mathcal{A}}\right) \leq V(\boldsymbol{x}) \leq \alpha_{2}\left(|\boldsymbol{x}|_{\mathcal{A}}\right) \\
\dot{V} \leq-\eta \alpha_{3}\left(|\boldsymbol{x}|_{\mathcal{A}}\right)+c .
\end{gathered}
$$

Then, the set

$$
\mathcal{B}:=\left\{\boldsymbol{x} \in \mathbb{R}^{q}:|\boldsymbol{x}|_{\mathcal{A}} \leq \alpha_{3}^{-1}(c / \eta)\right\}
$$

is uniformly globally asymptotically stable.

Consequently, $\mathcal{A}$ is uniformly globally practically asymptotically stable and (47) holds with $\delta:=\alpha_{3}^{-1}(c / \eta)$.

Remark 2 For simplicity in the notation, the bound (49) is taken to be linear in $\eta$. However, the statement holds as well if we replace $\eta$ by $\mu(\eta)$ for any $\mu \in \mathcal{K}_{\infty}$, with the appropriate modifications in the definitions of $\mathcal{B}$ and $\delta$.

In other words, Proposition 1 implies that a proper choice of $\eta$ in (46) ensures that the solutions come close to the set $\mathcal{A}$ with desired tolerance. For the particular case that $\mathcal{A}:=\{\boldsymbol{x}=0\}$ Proposition 1 implies uniform global practical asymptotic stability of the origin. Consequently, for any fixed $\eta$, we recover the property of global ultimate boundedness.

Everything is now in place to present our main statements on stability for networked systems with model (11) which, we recall, is equivalent to the equations

$$
\begin{aligned}
& \dot{\boldsymbol{e}}=-\sigma\left[L \otimes E_{m}\right] \boldsymbol{e}_{y}+\Pi\left[\tilde{F}\left(\boldsymbol{e}, \boldsymbol{x}_{s}\right)+F_{s}\left(\boldsymbol{x}_{s}\right)\right], \\
& \dot{\boldsymbol{x}}_{s}=f_{s}\left(\boldsymbol{x}_{s}\right)+G_{s}\left(\boldsymbol{e}, \boldsymbol{x}_{s}\right) .
\end{aligned}
$$

${ }^{5}$ That is, the solutions are defined on $\left[t_{0}, \infty\right)$. 
Firstly, in Section V-A, we analyze the behavior of the synchronization errors, that is, the stability properties of the sets $\mathcal{S}_{y}$ and $\mathcal{S}_{x}$, defined in (2) and (45). Next, in Section V-B, we analyze the behavior of the mean-field dynamics (51b). More precisely, we study the stability of a compact attractor which is proper to the emergent dynamics and we establish conditions under which the mean-field trajectories of the interconnected units remain close to this attractor.

\section{A. Network practical synchronization under diffusive coupling}

We formulate conditions that ensure practical global asymptotic stability of the sets $\mathcal{S}_{x}$ and $\mathcal{S}_{y}$. This implies practical state and output synchronization of the network, respectively. Furthermore, we show that the upper bound on the state synchronization error depends on the mismatches among the dynamics of the individual units of the network.

Theorem 1 (Output synchronization) Let the solutions of the system (11) be globally ultimately bounded with bound $B_{x}$ independent of $\sigma-$ see Def. 1. Then,

(i) the set $\mathcal{S}_{y}$ is uniformly globally practically asymptotically stable. In particular, for any $R>0$ and $\varepsilon>0$ there exists $T(R, \varepsilon)>0$ such that

$$
\left|\boldsymbol{e}_{y}\left(t, \boldsymbol{x}_{\circ}\right)\right| \leq \frac{C_{1}+C_{2}}{\sigma}\left|\mathcal{U}_{1}\right|+\varepsilon \quad \forall t \geq T, \boldsymbol{x}_{\circ} \in B_{R}
$$

where $C_{1}$ and $C_{2}$ are defined in (44).

(ii) If, moreover, the interconnection strength $\sigma$ is sufficiently large and Assumption 1 holds with the sets $\mathbb{B}_{z}:=\{z \in$ $\left.\mathbb{R}^{n-m}:|z| \leq B_{x}\right\}$ and $\mathbb{B}_{y}:=\left\{y \in \mathbb{R}^{m}:|y| \leq B_{x}\right\}$ then, the set

$\left\{\boldsymbol{x} \in \mathbb{R}^{n N}:|\boldsymbol{x}|_{\mathcal{S}_{x}} \leq c:=\frac{1}{\alpha_{m} N} \sqrt{N^{2} \Delta_{f}^{\prime}+\frac{C_{1}+C_{2}}{2}+\bar{\beta}}\right\}$

where $\Delta_{f}^{\prime}$ is a qualitative measure of the zero-dynamics's heterogeneity and

$$
\alpha_{m}:=\min _{1 \leq k \leq N} \bar{\alpha}_{k}, \quad \bar{\beta}=N \sum_{k=1}^{N} \beta_{k},
$$

is uniformly globally asymptotically stable.

Before presenting the proof of Theorem 1 (see farther below), we point out the following remarks. In words, the first statement implies that the output synchronization errors tend to a compact invariant set whose "size" depends on the heterogeneity of the network as well as on the synchronization errors relative to the solutions of the mean-field system, $\boldsymbol{x}_{s}(t)$, as well as on the ultimate bound on the trajectories, $B_{x}$ in (5) - see the inequalities in (44). Furthermore, the first statement establishes that the size of the residual set may be rendered arbitrarily small by augmenting the strength of the interconnection. That is, the property is robust vis a vis of the network's heterogeneity.

Also, observe that $|\boldsymbol{x}|_{\mathcal{S}_{x}}=|\boldsymbol{e}|$ hence, relying on a minimal interconnection strength, which is made explicit in the proof farther below, the second statement establishes that there exists an ultimate bound on the state-synchronization errors that depends on the heterogeneity of the network's zero-dynamics,
$\Delta_{f}^{\prime}$, on the ultimate bound on the trajectories, $B_{x}$, and on the constants $\bar{\alpha}_{k}$ and $\beta_{k}$ defined in Assumption 1. Under the assumptions of the theorem, however, this bound may not be diminished at will by increasing the interconnection gain $\sigma$.

Technically speaking, Statement (i) follows from two properties of the networked system -namely, negative definiteness of the second smallest eigenvalue of the Laplacian matrix $L$ and global ultimate boundedness of $\boldsymbol{x}(t)$.

As we establish next global ultimate boundedness holds under the following assumption.

Assumption 2 All the units (4) are strictly semi-passive with respect to the input $u_{i}$ and output $y_{i}$ with continuously differentiable and radially unbounded storage functions $V_{i}$ : $\mathbb{R}^{n} \rightarrow \mathbb{R}_{+}$, where $i \in \mathcal{I}$. That is, there exist positive definite and radially unbounded storage functions $V_{i}$, positive constants $\rho_{i}$, continuous functions $H_{i}$ and positive continuous functions $\psi_{i}$ such that

$$
\dot{V}_{i}\left(\boldsymbol{x}_{i}\right) \leq \boldsymbol{u}_{i}^{\top} \boldsymbol{y}_{i}-H_{i}\left(\boldsymbol{x}_{i}\right)
$$

and $H_{i}\left(\boldsymbol{x}_{i}\right) \geq \psi_{i}\left(\left|\boldsymbol{x}_{i}\right|\right)$ for all $\left|\boldsymbol{x}_{i}\right| \geq \rho_{i}$.

The following statement, for networks with directed strongly connected graphs, is reminiscent of [23, Corollary 1], which holds only for undirected graphs.

Proposition 2 Consider a network of $N$ diffusively coupled units (11). Let the graph of interconnections be undirected and connected and assume that all the units of the network are strictly semi-passive (i.e., Assumption 2 holds). Then, the solutions of the system (11) are ultimately bounded.

Proof: We follow similar steps as in the proofs of [23, Lemma 1] and [45, Proposition 2.1], where the case of undirected graphs was considered. Let Assumption 2 generate positive definite storage functions $V_{i}$ and let

$$
V_{\Sigma}(\boldsymbol{x}):=\sum_{i=1}^{N} \nu_{i} V_{i}\left(\boldsymbol{x}_{i}\right)
$$

where the constants $\nu_{i}$ correspond to the entries of $v_{\ell 1}$-see Eq. (12) (for undirected graphs, $\nu_{i}=1$ for all $i \leq N$ ). Since $\nu_{i}>0, V_{\Sigma}$ also qualifies as a storage function. Evaluating its total derivative along the trajectories of (4), and using (55), we obtain

$$
\dot{V}_{\Sigma}(\boldsymbol{x})=\sum_{i=1}^{N} \nu_{i} \boldsymbol{u}_{i}^{\top} \boldsymbol{y}_{i}-\sum_{i=1}^{N} \nu_{i} H_{i}\left(\boldsymbol{x}_{i}\right)
$$

Now, the first sum satisfies

$$
\sum_{i=1}^{N} \nu_{i} \boldsymbol{u}_{i}^{\top} \boldsymbol{y}_{i}=\boldsymbol{u}^{\top}\left[W \otimes I_{m}\right] \boldsymbol{y}
$$

where $W:=\operatorname{diag}\left\{\nu_{1}, \cdots, \nu_{N}\right\}$ hence, recalling that $\boldsymbol{u}=$ $-\sigma\left[L \otimes I_{m}\right]$, we obtain

$$
\begin{aligned}
\dot{V}_{\Sigma}(\boldsymbol{x}) & =-\sum_{i=1}^{N} \nu_{i} H_{i}\left(\boldsymbol{x}_{i}\right)-\sigma \boldsymbol{y}^{\top}\left[W \otimes I_{m}\right]\left[L \otimes I_{m}\right] \boldsymbol{y} \\
& =-\sum_{i=1}^{N} \nu_{i} H_{i}\left(\boldsymbol{x}_{i}\right)-\frac{1}{2} \sigma \boldsymbol{y}^{\top}\left[L_{s} \otimes I_{m}\right] \boldsymbol{y}
\end{aligned}
$$


where we used $W L \otimes I_{m}=\left[W \otimes I_{m}\right]\left[L \otimes I_{m}\right]$ and we defined $L_{s}:=W L+L^{\top} W$.

We show now that $L_{s}$ is positive semidefinite. To see this we observe first that $W$ is diagonal positive. In addition, the Laplacian $L$ has positive diagonal elements and non-positive off-diagonal elements hence, the same property holds for $W L$ and $L_{s}$. Furthermore, using the properties of $L$, that $L \mathbf{1}_{N}=0$, $v_{\ell 1}^{\top} L=0$, as well as the identity $W \mathbf{1}_{N}=v_{\ell 1}$, we see that

$$
L_{s} \mathbf{1}_{N}=\left(W L+L^{\top} W\right) \mathbf{1}_{N}=L^{\top} v_{\ell 1}=0 .
$$

That is, $\lambda_{1}=0$ is an eigenvalue of $L_{s}$ with corresponding eigenvector, $v_{\ell 1}=\mathbf{1}_{N}$. It follows that $L_{s}$ is a symmetric Laplacian matrix which satisfies $\boldsymbol{\xi}^{\top} L_{s} \boldsymbol{\xi} \geq 0$ for all $\boldsymbol{\xi} \in \mathbb{R}^{N}$ -cf. [7]. Thus, $\boldsymbol{y}^{\top}\left[L_{s} \otimes I_{m}\right] \boldsymbol{y} \geq 0$ for all $\boldsymbol{y} \in \mathbb{R}^{N m}$ and

$$
\dot{V}_{\Sigma}(\boldsymbol{x}) \leq-\sum_{i=1}^{N} \nu_{i} H_{i}\left(\boldsymbol{x}_{i}\right) .
$$

Next, let $\bar{\rho}=\max _{1 \leq i \leq N}\left\{\rho_{i}\right\}$ and consider the function $\bar{\psi}$ : $[\bar{\rho},+\infty) \rightarrow \mathbb{R}_{\geq 0}$ as $\bar{\psi}(s)=\min _{1 \leq i \leq N}\left\{\nu_{i} \rho_{i}(s)\right\}$. Since $\nu_{i}>0$ for all $i \leq N, \bar{\psi}$ is continuous and positive on its domain. Furthermore, we observe that, on one hand, by assumption $H_{i}\left(\boldsymbol{x}_{i}\right) \geq \psi_{i}\left(\left|\boldsymbol{x}_{i}\right|\right)$ for all $\boldsymbol{x}_{i}$ such that $\left|\boldsymbol{x}_{i}\right| \geq \rho_{i}$. On the other hand, for any $\boldsymbol{x} \in \mathbb{R}^{n N}$ such that $|\boldsymbol{x}| \geq N \bar{\rho}$ there exists $k \in \mathcal{I}$ such that $\left|\boldsymbol{x}_{k}\right| \geq \frac{1}{N}|\boldsymbol{x}| \geq \bar{\rho}$. Thus, for all $|\boldsymbol{x}| \geq N \bar{\rho}$ and any $k \leq N$,

$$
\sum_{i=1}^{N} \nu_{i} H_{i}\left(\boldsymbol{x}_{i}\right) \geq \nu_{k} H_{k}\left(\boldsymbol{x}_{k}\right) \geq \bar{\psi}\left(\left|\boldsymbol{x}_{k}\right|\right) \geq \bar{\psi}\left(\frac{1}{N}|\boldsymbol{x}|\right) .
$$

In view of the last bound above, the fact that $L_{s}$ is positive semidefinite (see farther below), and (57) we obtain, for all $|\boldsymbol{x}| \geq N \bar{\rho}$,

$$
\dot{V}_{\Sigma}(\boldsymbol{x}) \leq-\bar{\psi}\left(\frac{1}{N}|\boldsymbol{x}|\right) .
$$

Ultimate boundedness, of the solutions of the system (11), follows by invoking [46, Theorem 10.4].

Some interesting corollaries, on state synchronization, follow from Theorem 1. For instance, if the network interconnections depend on the whole state, that is, if $\boldsymbol{y}=\boldsymbol{x}$.

Corollary 1 Consider the system (11). Let Assumptions 1 and 2 be satisfied and let $\boldsymbol{y}=\boldsymbol{x}$. Then, the system is forward complete and the set $\mathcal{S}_{x}$ is uniformly globally practically asymptotically stable.

Proof: By Assumption 2, Proposition 2 holds so the system is forward complete and, moreover, the solutions are globally ultimately bounded. Therefore, the statement follows straightforward by invoking Theorem 1 with the output $\boldsymbol{y}=\boldsymbol{x}$ and $\boldsymbol{e}_{y}=\boldsymbol{x}-\mathbf{1}_{N} \otimes \boldsymbol{x}_{s}=\boldsymbol{e}$.

The constant $\Delta_{f}^{\prime}$ represents the maximal possible mismatch between the dynamics of any individual unit and that of the mean-field unit, on a ball of radius $B_{x}$. The more heterogeneous the network is, the bigger is the constant $\Delta_{f}^{\prime}$. Conversely, in the case that all the zero dynamics of the units are identical, we have $\Delta_{f}^{\prime}=0$. In this case, we directly obtain the following statement from (53).

Corollary 2 Consider the system (11) under Assumptions 1 and 2. Assume, further, that the zero dynamics of the network units are all identical (i.e., $f_{i}^{z}(x)=f_{j}^{z}(x)$ for all $i, j \in \mathcal{I}$ and all $x \in \mathbb{R}^{n}$ ). Then, the set $\mathcal{S}_{x}$ is uniformly globally practically asymptotically stable.

\section{Proof of Theorem 1}

Let $R>0$ be arbitrary. Since, by assumption, the solutions are uniformly ultimately bounded, for any $\boldsymbol{x}_{\circ} \in B_{R}$ there exists $T_{1}(R)>0$ such that $\left|\boldsymbol{x}\left(t, \boldsymbol{x}_{\circ}\right)\right| \leq B_{x}$ for all $t \geq T_{1}$. In the sequel, we consider the behavior of the solutions for $t \geq T_{1}$.

Proof of $(i)$. The proof relies on Proposition 1. By assumption, the solutions are globally ultimately bounded hence, the system is forward complete. Then, let us introduce the function $V_{y}: \mathbb{R}^{m N} \rightarrow \mathbb{R}_{+}$defined, with an abuse of notation, as

$$
V_{y}(\boldsymbol{x})=\boldsymbol{e}_{y}^{\top}\left[\Gamma \otimes I_{m}\right] \boldsymbol{e}_{y}, \quad \Gamma:=\left(U^{-1}\right)^{*} \mathcal{P} U^{-1}
$$

where $\mathcal{P}$ is defined in (72)-see Appendix A. Indeed, note that $\boldsymbol{e}_{y}$ is a function of $\boldsymbol{x}$ since

$$
\boldsymbol{e}_{y}=\left[I_{N} \otimes E_{m}^{\top}\right] \boldsymbol{e}
$$

and, after (21),

$$
\boldsymbol{e}=\left[I_{n N}-\left(\mathbf{1}_{N} v_{\ell 1}^{\top}\right) \otimes I_{n}\right] \boldsymbol{x}
$$

Therefore, $\left|\boldsymbol{e}_{y}\right|=|\boldsymbol{x}|_{\mathcal{S}_{y}}$ and, consequently, $V_{y}(\boldsymbol{x})$ trivially satisfies (48) with $\mathcal{A}=\mathcal{S}_{y}, \alpha_{i}(s):=c_{i} s^{2}$.

Now, differentiating on both sides of (59) and using (51a), we obtain

$$
\begin{aligned}
\dot{\boldsymbol{e}}_{y}= & -\sigma\left(I_{N} \otimes E_{m}^{\top}\right)\left(L \otimes E_{m}\right) \boldsymbol{e}_{y} \\
& +\left(I_{N} \otimes E_{m}^{\top}\right)\left[\Pi \tilde{F}\left(\boldsymbol{e}, \boldsymbol{x}_{s}\right)+\Pi F_{s}\left(\boldsymbol{x}_{s}\right)\right] .
\end{aligned}
$$

Hence, differentiating $V_{y}(\boldsymbol{x})$ along trajectories of (60), using the identities $\left(I_{N} \otimes E_{m}^{\top}\right)\left(L \otimes E_{m}\right)=L \otimes\left(E_{m}^{\top} E_{m}\right)=L \otimes I_{m}$ and the triangle inequality, we obtain

$$
\begin{aligned}
\dot{V}_{y}(\boldsymbol{x})=- & \sigma \boldsymbol{e}_{y}^{\top}\left[\Gamma \otimes I_{m}\right]\left[L \otimes I_{m}\right] \boldsymbol{e}_{y} \\
- & \sigma \boldsymbol{e}_{y}^{\top}\left[L^{\top} \otimes I_{m}\right]\left[\Gamma \otimes I_{m}\right] \boldsymbol{e}_{y} \\
& +\left[\left|\Pi \tilde{F}\left(\boldsymbol{e}, \boldsymbol{x}_{s}\right)\right|+\left|\Pi F_{s}\left(\boldsymbol{x}_{s}\right)\right|\right]\left|\boldsymbol{e}_{y}\right| \\
=- & \sigma \boldsymbol{e}_{y}^{\top}\left[\left(\Gamma L-L^{\top} \Gamma\right) \otimes I_{m}\right] \boldsymbol{e}_{y} \\
+ & +\left[\left|\Pi \tilde{F}\left(\boldsymbol{e}, \boldsymbol{x}_{s}\right)\right|+\left|\Pi F_{s}\left(\boldsymbol{x}_{s}\right)\right|\right]\left|\boldsymbol{e}_{y}\right| .
\end{aligned}
$$

Therefore, proceeding as in the proof of Lemma 1 and redefining $c_{U}:=\left|\mathcal{U}_{1}\right|$, we obtain

$$
\dot{V}_{y}(\boldsymbol{x}) \leq-\sigma c_{U}\left|\boldsymbol{e}_{y}\right|^{2}+\left[\left|\Pi \tilde{F}\left(\boldsymbol{e}, \boldsymbol{x}_{s}\right)\right|+\left|\Pi F_{s}\left(\boldsymbol{x}_{s}\right)\right|\right]\left|\boldsymbol{e}_{y}\right|,
$$

which, in view of (44), implies that

$$
\dot{V}_{y}(\boldsymbol{x}) \leq-\sigma c_{U}\left|\boldsymbol{e}_{y}\right|^{2}+C\left|\boldsymbol{e}_{y}\right| \quad \forall t \geq T_{1}
$$

where $C:=C_{1}+C_{2}$. Notice that the constants $C_{1}$ and $C_{2}$ depend only on the functions $\tilde{F}, F_{s}$ and on the constant $B_{x}$ and are independent of $\sigma$. Then, for any $\sigma>0$,

$$
\dot{V}_{y}(\boldsymbol{x}) \leq-\frac{1}{2} \sigma c_{U}\left|\boldsymbol{e}_{y}\right|^{2}+\frac{C^{2}}{2 \sigma} .
$$

Therefore, (49) holds with $\eta=\sigma, \alpha_{3}(s)=c_{U} s^{2} / 2$ and $c:=$ $C^{2} / 2 \sigma$. The result follows invoking Proposition 1 . 
Proof of (ii). Let $\mathcal{B}_{B_{x}}:=\left\{\boldsymbol{z} \in \mathbb{R}^{(n-m) N}:|\boldsymbol{z}| \leq B_{x}\right\}$ that is, $\mathcal{B}_{B_{x}} \subset \mathbb{B}_{z}^{N}$. Let Assumption 1 generate functions $V_{\circ k}$, with $k \leq N$, and let us consider the continuously differentiable function $V_{z}: \mathcal{B}_{B_{x}} \rightarrow \mathbb{R}_{+}$, defined as

$$
V_{z}(\boldsymbol{z})=\sum_{k=1}^{N} \sum_{i=1}^{N} V_{\circ k}\left(\boldsymbol{z}_{k}-\boldsymbol{z}_{i}\right) .
$$

For the sequel, we make the following technical statement.

Claim 1 There exist functions $\gamma_{1}, \gamma_{2} \in \mathcal{K}_{\infty}$ such that $V_{z}$ satisfies the bounds

$$
\gamma_{1}\left(\left|\boldsymbol{e}_{z}\right|\right) \leq V_{z}(\boldsymbol{z}) \leq \gamma_{2}\left(\left|\boldsymbol{e}_{z}\right|\right)
$$

where $\boldsymbol{e}_{z}:=\left[I_{N} \otimes E_{n-m}^{\top}\right] \boldsymbol{e}=\boldsymbol{z}-\mathbf{1}_{N} \otimes \boldsymbol{z}_{s}$.

Proof of Claim 1. In view of Assumption 1, there exist a set $\mathbb{B}_{z} \subset \mathbb{R}^{n-m}$ and, for each $k \leq N$, there exist class $\mathcal{K}_{\infty}$ functions $\gamma_{1 k}, \gamma_{2 k}$ such that the functions $V_{\circ k}$ satisfy

$$
\gamma_{1 k}\left(\left|\boldsymbol{z}_{k}-\boldsymbol{z}_{i}\right|\right) \leq V_{\circ k}\left(\boldsymbol{z}_{k}-\boldsymbol{z}_{i}\right) \leq \gamma_{2 k}\left(\left|\boldsymbol{z}_{k}-\boldsymbol{z}_{i}\right|\right)
$$

for all $\boldsymbol{z}_{i}, \boldsymbol{z}_{k} \in \mathbb{B}_{z}$. Hence,

$$
V_{z}(\boldsymbol{z}) \geq \sum_{k=1}^{N} \sum_{i=1}^{N} \gamma_{1 k}\left(\left|\boldsymbol{z}_{k}-\boldsymbol{z}_{i}\right|\right) .
$$

Now, let $\gamma_{m}(s):=\min _{1 \leq k \leq N}\left\{\gamma_{1 k}(s)\right\}$; since the functions $\gamma_{1 k} \in \mathcal{K}_{\infty}$ for all $k \leq \bar{N}, \gamma_{m}$ is continuous and of class $\mathcal{K}_{\infty}$. Therefore,

$$
V_{z}(\boldsymbol{z}) \geq \sum_{k=1}^{N} \sum_{i=1}^{N} \gamma_{m}\left(\left|\boldsymbol{z}_{k}-\boldsymbol{z}_{i}\right|\right) .
$$

Then, let us define $\gamma_{m}^{\prime}(s):=\gamma_{m}(\sqrt{s})$, which is also of class $\mathcal{K}_{\infty}$ hence, after Lemma 2 from Appendix B on p. 15, we obtain

$$
V_{z}(\boldsymbol{z}) \geq \sum_{k=1}^{N} \gamma_{m}^{\prime}\left(\frac{1}{N} \sum_{i=1}^{N}\left|\boldsymbol{z}_{k}-\boldsymbol{z}_{i}\right|^{2}\right)
$$

Next, we invoke once more Lemma 2 and, successively, Lemma 3 from Appendix B to obtain

$$
\begin{aligned}
V_{z}(\boldsymbol{z}) & \left.\geq \gamma_{m}^{\prime}\left(\frac{1}{N^{2}} \sum_{k=1}^{N} \sum_{i=1}^{N}\left|\boldsymbol{z}_{k}-\boldsymbol{z}_{i}\right|^{2}\right)\right) \\
& \geq \gamma_{m}^{\prime}\left(\frac{\left|\boldsymbol{z}-\mathbf{1}_{N} \otimes \boldsymbol{z}_{s}\right|^{2}}{N^{2}\left|v_{\ell 1} v_{\ell 1}^{\top}\right|_{2}}\right)=\gamma_{m}^{\prime}\left(\frac{\left|\boldsymbol{e}_{z}\right|^{2}}{N^{2}\left|v_{\ell 1} v_{\ell 1}^{\top}\right|_{2}}\right)
\end{aligned}
$$

so, defining ${ }^{6} c_{v}:=N^{2}\left|v_{\ell 1} v_{\ell 1}^{\top}\right|_{2}$, we see that the lower-bound in (63) follows with

$$
\gamma_{1}(s):=\gamma_{m}^{\prime}\left(\frac{s^{2}}{c_{v}}\right)
$$

which is of class $\mathcal{K}_{\infty}$. The existence of $\gamma_{2} \in \mathcal{K}_{\infty}$, such that $V_{z}(\boldsymbol{z}) \leq \gamma_{2}\left(\left|\boldsymbol{e}_{z}\right|\right)$ is deduced following similar arguments.

Next, we evaluate the total derivative of $V_{z}(\boldsymbol{z})$ along trajectories of the system (4b), to obtain

$$
\dot{V}_{z}(\boldsymbol{z})=\sum_{k=1}^{N} \sum_{i=1}^{N} \dot{V}_{\circ k}\left(\boldsymbol{z}_{k}-\boldsymbol{z}_{i}\right)
$$

\footnotetext{
${ }^{6}$ For a matrix $M \in \mathbb{R}^{N \times N},|M|_{2}$ denotes the induced 2-norm that is, $|M|_{2}:=\max _{\boldsymbol{z} \neq 0} \frac{\boldsymbol{z}^{\top} M \boldsymbol{z}}{\boldsymbol{z}^{\top} \boldsymbol{z}}$.
}

where, using Assumption 1, we obtain

$$
\begin{aligned}
\dot{V}_{\circ k}\left(\boldsymbol{z}_{k}-\boldsymbol{z}_{i}\right)= & \nabla V_{\circ k}\left(\boldsymbol{z}_{k}-\boldsymbol{z}_{i}\right)\left[f_{k}^{z}\left(\boldsymbol{y}_{k}, \boldsymbol{z}_{k}\right)-f_{i}^{z}\left(\boldsymbol{y}_{i}, \boldsymbol{z}_{i}\right)\right] \\
= & \nabla V_{\circ k}\left(\boldsymbol{z}_{k}-\boldsymbol{z}_{i}\right)\left[f_{k}^{z}\left(\boldsymbol{y}_{k}, \boldsymbol{z}_{k}\right)-f_{k}^{z}\left(\boldsymbol{y}_{i}, \boldsymbol{z}_{k}\right)\right. \\
& \left.\quad+f_{k}^{z}\left(\boldsymbol{y}_{i}, \boldsymbol{z}_{k}\right)-f_{i}^{z}\left(\boldsymbol{y}_{i}, \boldsymbol{z}_{i}\right)\right] \\
\leq & -\bar{\alpha}_{k}\left|\boldsymbol{z}_{k}-\boldsymbol{z}_{i}\right|^{2}+\beta_{k} \\
& +\nabla V_{\circ k}\left(\boldsymbol{z}_{k}-\boldsymbol{z}_{i}\right)\left[f_{k}^{z}\left(\boldsymbol{y}_{i}, \boldsymbol{z}_{k}\right)-f_{k}^{z}\left(\boldsymbol{y}_{i}, \boldsymbol{z}_{i}\right)\right] \\
& +\nabla V_{\circ k}\left(\boldsymbol{z}_{k}-\boldsymbol{z}_{i}\right)\left[f_{k}^{z}\left(\boldsymbol{y}_{i}, \boldsymbol{z}_{i}\right)-f_{i}^{z}\left(\boldsymbol{y}_{i}, \boldsymbol{z}_{i}\right)\right] .
\end{aligned}
$$

Now, in view of the continuity of $\nabla V_{\circ k}$, there exists a constant $C_{\circ}>0$ such that for all $|\boldsymbol{x}| \leq B_{x}$ we have, for all $k \in \mathcal{I}$,

$$
\left|\nabla V_{\circ k}\left(\boldsymbol{z}_{k}-\boldsymbol{z}_{i}\right)\right| \leq C_{\circ}\left|\boldsymbol{z}_{k}-\boldsymbol{z}_{i}\right| \text {. }
$$

Also, since the functions $f_{i}^{z}$ are locally Lipschitz, there exists a continuous, positive, non-decreasing function $k_{z}: \mathbb{R}_{+} \rightarrow \mathbb{R}_{+}$ such that for all $k \in \mathcal{I}$,

$$
\left|f_{k}^{z}\left(\boldsymbol{y}_{i}, \boldsymbol{z}_{k}\right)-f_{k}^{z}\left(\boldsymbol{y}_{i}, \boldsymbol{z}_{i}\right)\right| \leq k_{z}(|\boldsymbol{z}|)\left|\boldsymbol{y}_{k}-\boldsymbol{y}_{i}\right|
$$

and for all $|\boldsymbol{x}| \leq B_{x}$, hence all $|\boldsymbol{z}| \leq B_{x}$,

$$
\begin{gathered}
\left|f_{k}^{z}\left(\boldsymbol{y}_{i}, \boldsymbol{z}_{k}\right)-f_{k}^{z}\left(\boldsymbol{y}_{i}, \boldsymbol{z}_{i}\right)\right| \leq k_{z}\left(B_{x}\right)\left|\boldsymbol{y}_{k}-\boldsymbol{y}_{i}\right|=: C_{z}\left|\boldsymbol{y}_{k}-\boldsymbol{y}_{i}\right| \\
=C_{z}\left|\boldsymbol{y}_{k}-\boldsymbol{y}_{s}+\boldsymbol{y}_{s}-\boldsymbol{y}_{i}\right| \\
\leq C_{z}\left(\left|\boldsymbol{y}_{k}-\boldsymbol{y}_{s}\right|+\left|\boldsymbol{y}_{s}-\boldsymbol{y}_{i}\right|\right) \\
=2 C_{z}\left|\boldsymbol{e}_{y}\right|
\end{gathered}
$$

Furthermore, in view of the continuity of $f_{i}^{z}$, we may also define

$$
\Delta_{f}:=\max _{|\boldsymbol{x}| \leq B_{x}} \max _{k, i \in N}\left\{\left|f_{k}^{z}\left(\boldsymbol{y}_{i}, \boldsymbol{z}_{i}\right)-f_{i}^{z}\left(\boldsymbol{y}_{i}, \boldsymbol{z}_{i}\right)\right|\right\} .
$$

Therefore, using all the previous bounds in (65) we obtain, for all $i, k \leq N$ and all $\boldsymbol{z}_{k} \in \mathbb{B}_{z k}$,

$$
\begin{aligned}
\dot{V}_{\circ k}\left(\boldsymbol{z}_{k}-\boldsymbol{z}_{i}\right) \leq & -\bar{\alpha}_{k}\left|\boldsymbol{z}_{k}-\boldsymbol{z}_{i}\right|^{2} \\
& +2 C_{\circ}\left|\boldsymbol{z}_{k}-\boldsymbol{z}_{i}\right|\left(C_{z}\left|\boldsymbol{e}_{y}\right|+\Delta_{f}\right)+\beta_{k} \\
\leq & -\frac{1}{2} \bar{\alpha}_{k}\left|\boldsymbol{z}_{k}-\boldsymbol{z}_{i}\right|^{2}+\bar{C}\left|\boldsymbol{e}_{y}\right|^{2}+\Delta_{f}^{\prime}+\beta_{k},
\end{aligned}
$$

where

$$
\bar{C}=\frac{2 C_{\circ}^{2} C_{z}^{2}}{\alpha_{m}}, \quad \Delta_{f}^{\prime}=\frac{2 C_{\circ}^{2} \Delta_{f}^{2}}{\alpha_{m}} .
$$

In turn, it follows that

$$
\begin{aligned}
\dot{V}_{z}(\boldsymbol{z}) \leq & -\sum_{k=1}^{N} \sum_{i=1}^{N}\left(\frac{1}{2} \bar{\alpha}_{k}\left|\boldsymbol{z}_{k}-\boldsymbol{z}_{i}\right|^{2}-\bar{C}\left|\boldsymbol{e}_{y}\right|^{2}-\Delta_{f}^{\prime}+\beta_{k}\right) \\
\leq & -N \alpha_{m}\left|\boldsymbol{z}-\mathbf{1}_{N} \otimes \boldsymbol{z}_{s}\right|^{2}+\bar{C} N^{2}\left|\boldsymbol{e}_{y}\right|^{2} \\
& \quad+N^{2} \Delta_{f}^{\prime}+\sum_{k=1}^{N} N \beta_{k} \\
\leq & -N \alpha_{m}\left|\boldsymbol{e}_{z}\right|^{2}+\bar{C} N^{2}\left|\boldsymbol{e}_{y}\right|^{2}+N^{2} \Delta_{f}^{\prime}+\bar{\beta}
\end{aligned}
$$

where, for the second step, we invoked Lemma 3 from Appendix B.

Finally, we define $V_{e}(\boldsymbol{x}):=V_{y}(\boldsymbol{x})+V_{z}(\boldsymbol{z})$ which, in view of Claim 1, satisfies

$$
\frac{1}{2}\left|\boldsymbol{e}_{y}\right|^{2}+\gamma_{1}\left(\left|\boldsymbol{e}_{z}\right|\right) \leq V_{e}(\boldsymbol{x}) \leq \frac{1}{2}\left|\boldsymbol{e}_{y}\right|^{2}+\gamma_{2}\left(\left|\boldsymbol{e}_{z}\right|\right),
$$


which is equivalent to $\tilde{\gamma}_{1}(|\boldsymbol{e}|) \leq V_{e}(\boldsymbol{x}) \leq \tilde{\gamma}_{2}(|\boldsymbol{e}|)$ for an appropriate choice of $\tilde{\gamma}_{1}, \tilde{\gamma}_{2} \in \mathcal{K}_{\infty}$. Hence, (48) holds with $\mathcal{A}=\mathcal{S}_{x}$.

On the other hand, from (61) and (67), we obtain $\dot{V}_{e}(\boldsymbol{x}) \leq-\alpha_{m} N\left|\boldsymbol{e}_{z}\right|^{2}-\frac{1}{2}\left[\sigma c_{U}-2 \bar{C} N^{2}\right]\left|\boldsymbol{e}_{y}\right|^{2}+N^{2} \Delta_{f}^{\prime}+\frac{C^{2}}{2 \sigma}+\bar{\beta}$. Therefore, there exists $\sigma^{*}>0$ such that for all $\sigma \geq \sigma^{*}$,

$$
\dot{V}_{e}(\boldsymbol{x}) \leq-\alpha_{m} N|\boldsymbol{e}|^{2}+N^{2} \Delta_{f}^{\prime}+\frac{C^{2}}{2 \sigma^{*}}+\bar{\beta} .
$$

The proof is completed invoking Proposition 1 with fixed $\eta:=$ $\alpha_{m} N, \alpha_{3}(s)=s^{2}$ and $c=N^{2} \Delta_{f}^{\prime}+C^{2} / 2 \sigma^{*}+\bar{\beta}$.

Remark 3 In general, the quantities $C_{1}$ and $C_{2}$ in the ultimate bound (53) may grow non-linearly, relatively to the size of the network and independently of the strength of the interconnection $\sigma$. However, in the particular case of identical zero-dynamics ( $c f$. Corollary 2) the eventual influence of the size of the network, on the size of the residual set may be compensated by the interconnection strength, up to the limit $\varepsilon-$ see $(52)$.

\section{B. On practical stability of the collective network behavior}

Now we analyze the behavior of the mean-field unit, whose dynamics is given by the equations $(51 \mathrm{~b})$. We assume that the nominal dynamics of the latter (i.e., with $e=0$ ) has a stable compact attractor $\mathcal{A}$ and we establish that the stability properties of this attractor are preserved under the network interconnection, albeit, slightly weakened.

Assumption 3 For the emergent dynamics, (28), there exists a compact invariant set $\mathcal{A} \subset \mathbb{R}^{n}$ which is asymptotically stable with a domain of attraction $\mathcal{D} \subset \mathbb{R}^{n}$. Moreover, we assume that there exists a continuously differentiable Lyapunov function $V_{\mathcal{A}}: \mathbb{R}^{n} \rightarrow \mathbb{R}_{\geq 0}$ and functions $\alpha_{i} \in \mathcal{K}_{\infty}, i \in\{1, \ldots, 4\}$ such that for all $\boldsymbol{x}_{e} \in \mathcal{D}$ we have

$$
\begin{gathered}
\alpha_{1}\left(\left|\boldsymbol{x}_{e}\right|_{\mathcal{A}}\right) \leq V_{\mathcal{A}}\left(\boldsymbol{x}_{e}\right) \leq \alpha_{2}\left(\left|\boldsymbol{x}_{e}\right|_{\mathcal{A}}\right) \\
\dot{V}_{\mathcal{A}}\left(\boldsymbol{x}_{e}\right) \leq-\alpha_{3}\left(\left|\boldsymbol{x}_{e}\right|_{\mathcal{A}}\right) \\
\left|\frac{\partial V_{\mathcal{A}}}{\partial \boldsymbol{x}_{e}}\right| \leq \alpha_{4}\left(\left|\boldsymbol{x}_{e}\right|\right) .
\end{gathered}
$$

The second part of the assumption (the existence of $V$ ) is purely technical whereas the first part is essential to analyze the emergent synchronized behavior as well as the synchronization properties of the network, recasted as a (robust) stability problem. The following statement applies to the general case of diffusively coupled networks.

Theorem 2 For the system (11) let us assume that the solutions are globally ultimately bounded and Assumptions 1 and 3 hold. Then, there exists $B_{x s}>0$, such that the set

$$
\mathcal{B}:=\left\{\boldsymbol{x} \in \mathbb{R}^{q}:|\boldsymbol{x}|_{\mathcal{A}} \leq \alpha_{3}^{-1}\left(\alpha_{4}\left(B_{x}\right) k\left(c, B_{x s}\right)\right)\right\}
$$

where $k$ is defined in (34) and $c$ is defined in (53), is uniformly asymptotically stable.

Proof: Global ultimate boundedness of the solutions implies that there exists $T^{*}>0$ such that $\left|\boldsymbol{x}\left(t, \boldsymbol{x}_{\circ}\right)\right| \leq B_{x}$ for all $t \geq T^{*}$; therefore, a similar bound is valid for $\boldsymbol{x}_{s}$ :

$$
\left|\boldsymbol{x}_{s}\left(t, \boldsymbol{x}_{\circ}\right)\right|=\left|\sum_{i=1}^{N} \nu_{i} \boldsymbol{x}_{i}\left(t, \boldsymbol{x}_{\circ}\right)\right| \leq \sum_{i=1}^{N}\left|\nu_{i} \boldsymbol{x}_{i}\left(t, \boldsymbol{x}_{\circ}\right)\right| \leq B_{x s} .
$$

This and Assumption 1 imply the statement (ii) of Theorem 1. Hence, using (34) we obtain $\left|G_{s}\left(\boldsymbol{e}(t), \boldsymbol{x}_{s}(t)\right)\right| \leq B_{g}$ where

$$
B_{g}:=k\left(c, B_{x s}\right) c .
$$

Next, let Assumption 3 generate a Lyapunov function $V_{\mathcal{A}}$ for the nominal system (27) and functions $\alpha_{i} \in \mathcal{K}_{\infty}$, satisfying (68), hence, for all $\boldsymbol{x}_{s} \in \mathcal{D}$,

$$
\begin{gathered}
\alpha_{1}\left(\left|\boldsymbol{x}_{s}\right|_{\mathcal{A}}\right) \leq V_{\mathcal{A}}\left(\boldsymbol{x}_{s}\right) \leq \alpha_{2}\left(\left|\boldsymbol{x}_{s}\right|_{\mathcal{A}}\right) \\
\dot{V}_{\mathcal{A}}\left(\left|\boldsymbol{x}_{s}\right|_{\mathcal{A}}\right) \leq-\alpha_{3}\left(\left|\boldsymbol{x}_{s}\right|_{\mathcal{A}}\right) \\
\left|\nabla V_{\mathcal{A}}\left(\boldsymbol{x}_{s}\right)\right| \leq \alpha_{4}\left(\left|\boldsymbol{x}_{s}\right|\right)
\end{gathered}
$$

and the total derivative of $V_{\mathcal{A}}$ yields, using (33),

$$
\dot{V}_{\mathcal{A}}\left(\boldsymbol{x}_{s}\right) \leq-\alpha_{3}\left(\left|\boldsymbol{x}_{s}\right|_{\mathcal{A}}\right)+\alpha_{4}\left(\left|\boldsymbol{x}_{s}\right|_{\mathcal{A}}\right)\left|G_{s}\left(\boldsymbol{e}, \boldsymbol{x}_{s}\right)\right|
$$

which implies that

$$
\dot{V}_{\mathcal{A}}\left(\boldsymbol{x}_{s}(t)\right) \leq-\alpha_{3}\left(\left|\boldsymbol{x}_{s}(t)\right|_{\mathcal{A}}\right)+\alpha_{4}\left(B_{x s}\right) B_{g}
$$

for all $\boldsymbol{x}_{\circ}$ such that $\boldsymbol{x}_{s}(t) \in \mathcal{D}$. Strictly speaking, the bound (71) holds for all $t \geq T^{*}$ however, in view of forward completeness, the trajectories are bounded on $\left[0, T^{*}\right]$ therefore, (71) holds for all $t \geq 0$ by redefining, if necessary, the constants $B_{x s}$ and $B_{g}$ in function of $R$ i.e., of the ball of initial conditions. The result follows after Proposition 1.

In the case that the network is state practically synchronized, it follows that the set $\mathcal{A}$ is practically stable for the network (11).

Corollary 3 Consider the system (11) under Assumption 3. If the set $\mathcal{S}_{x}$ is uniformly globally practically asymptotically stable for this system, then the attractor $\mathcal{A}$ defined in Assumption 3 is practically asymptotically stable for the mean-field unit (33).

\section{EXAMPLE}

To illustrate our theoretical findings we present a brief casestudy of analysis of interconnected heterogeneous systems via diffusive coupling. We consider three chaotic oscillators, two of the well-known Lorenz type -see [47], with different parameters, and a Lü system [48]. The dynamics equations are

$$
\begin{aligned}
& \text { LORENZ: }\left\{\begin{array}{l}
\dot{x}_{i}=\gamma_{i}\left(y_{i}-x_{i}\right)+u_{i}, \quad i=1,2 \\
\dot{y}_{i}=r_{i} x_{i}-y_{i}-x_{i} z_{i} \\
\dot{z}_{i}=x_{i} y_{i}-b_{i} z_{i}
\end{array}\right. \\
& \text { L } \ddot{\mathrm{U}}:\left\{\begin{array}{l}
\dot{x}_{3}=-\frac{\alpha \beta}{\alpha+\beta} x_{3}-2 y_{3} z_{3}+\delta+u_{3} \\
\dot{y}_{3}=\alpha y_{3}+x_{3} z_{3} \\
\dot{z}_{3}=\beta z_{3}+x_{3} y_{3} .
\end{array}\right.
\end{aligned}
$$

The values of the parameters of the three systems are fixed in order for them to exhibit a chaotic behavior: 


\begin{tabular}{|c|c|c|}
\hline$\gamma_{1}=10$ & $\gamma_{2}=16$ & $\alpha=-10$ \\
\hline$r_{1}=45.6$ & $r_{2}=99.96$ & $\beta=-4$ \\
\hline$b_{1}=4$ & $b_{2}=8 / 3$ & $\delta=10$ \\
\hline
\end{tabular}

A direct computation, using (25), shows that the corresponding emergent dynamics for these systems is given by

$$
\begin{aligned}
& \text { EMERGENT } \\
& \text { DYNAMICS: }
\end{aligned}\left\{\begin{aligned}
3 \dot{x}_{e}= & -\left[\gamma_{1}+\gamma_{2}+\frac{\alpha \beta}{\alpha+\beta}\right] x_{e} \\
& +\left[\gamma_{1}+\gamma_{2}-2 z_{e}\right] y_{e}+\delta, \\
3 \dot{y}_{e}= & {\left[r_{1}+r_{2}\right] x_{e}-[2-\alpha] y_{e} } \\
3 \dot{z}_{e}= & 3 x_{e} y_{e}+\left[\beta-b_{1}-b_{2}\right] z_{e} .
\end{aligned}\right.
$$

Since the three chaotic systems are oscillators their trajectories are globally ultimately bounded -see Figure 3. Moreover, as it may be appreciated from Figure 4, the solutions remain bounded under the diffusive coupling which, for this test, we defined to be:

$$
\begin{array}{lll}
u_{1}=-\sigma\left[a_{12}\left(x_{1}-x_{2}\right)+a_{13}\left(x_{1}-x_{3}\right)\right], & a_{12}=2, \\
u_{2}=-\sigma\left[a_{21}\left(x_{2}-x_{1}\right)+a_{23}\left(x_{2}-x_{3}\right)\right], & a_{13}=4, \\
u_{3}=-\sigma\left[a_{31}\left(x_{3}-x_{1}\right)+a_{32}\left(x_{3}-x_{2}\right)\right], & a_{23}=3 .
\end{array}
$$

That is, the zero dynamics with respect to the output $\boldsymbol{y}_{i}=x_{i}$ has dimension two. For each Lorenz system, the zero dynamics is practically convergent (Assumption 1 holds), as it may be showed using the function

$$
V\left(\boldsymbol{z}_{i}-\boldsymbol{z}_{i}^{\prime}\right)=\left|\boldsymbol{z}_{i}-\boldsymbol{z}_{i}^{\prime}\right|^{2}, \quad \boldsymbol{z}_{i}=\left[y_{i} z_{i}\right]^{\top}, \quad i \in\{1,2\} .
$$

whose total derivative yields

$$
\dot{V}\left(\boldsymbol{z}_{i}-\boldsymbol{z}_{i}^{\prime}\right) \leq-2 \min \left\{b_{i}, 2\right\}\left|\boldsymbol{z}_{i}-\boldsymbol{z}_{i}^{\prime}\right|^{2} .
$$

For the Lü system, we have, defining $\boldsymbol{z}=\left[y_{3} z_{3}\right]^{\top}$,

$$
\begin{aligned}
\dot{V}\left(\boldsymbol{z}-\boldsymbol{z}^{\prime}\right) \leq- & -2 \alpha\left|y_{3}-y_{3}^{\prime}\right|^{2}-2 \beta\left|z_{3}-z_{3}^{\prime}\right|^{2} \\
& +4\left|x_{3}(t)\right|\left|y_{3}(t)-y_{3}^{\prime}(t) \| z_{3}(t)-z_{3}^{\prime}(t)\right| .
\end{aligned}
$$

Convergence in a practical sense (Assumption 1) holds since the trajectories are ultimately bounded hence, so is the last term on the right-hand side of the previous inequality.

Simulation results for different values of the interconnection gain $\sigma$ are showed in Figure 4; it may be appreciated that the the output synchronization errors, $\boldsymbol{e}_{y}(t)$, diminish as the interconnection gain is increased. The phase portraits of the three oscillators and that of the mean-field dynamics, are also showed for three different values of $\sigma$.

In Figure 5 we show the phase portrait of the mean-field dynamics (51b), for different values of the interconnection gain, compared to that of the emergent dynamics (28). As it is appreciated, the solutions generated by the emergent dynamics converge to an equilibrium -approximately, the point $(2.9,29.43)$ that is, in this case the attractor $\mathcal{A}$, defined in Assumption 3, is a point in the phase space. The mean-field trajectories converge to a double scroll attractor for "small" values of $\sigma$ and to a point in the space, at short distance from $\mathcal{A}$; roughly for $\sigma>15$. This illustrates that the emergent dynamics constitutes, to some extent, a good "estimate" of the network's collective behavior.
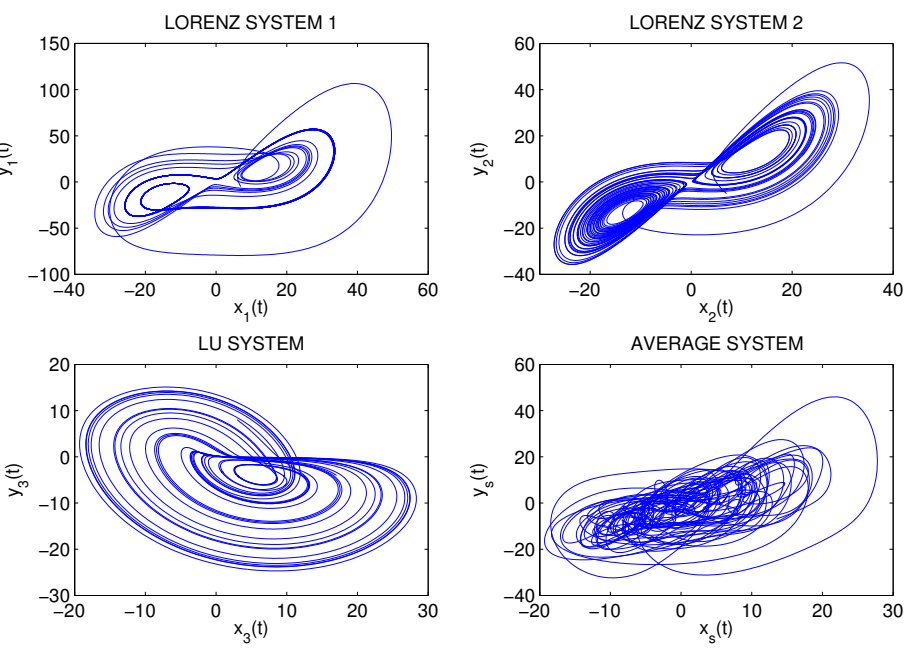

Fig. 3. Phase portraits of the three chaotic oscillators as well as that of the mean-field dynamics, in the absence of interconnection, i.e., with $\sigma=0$.
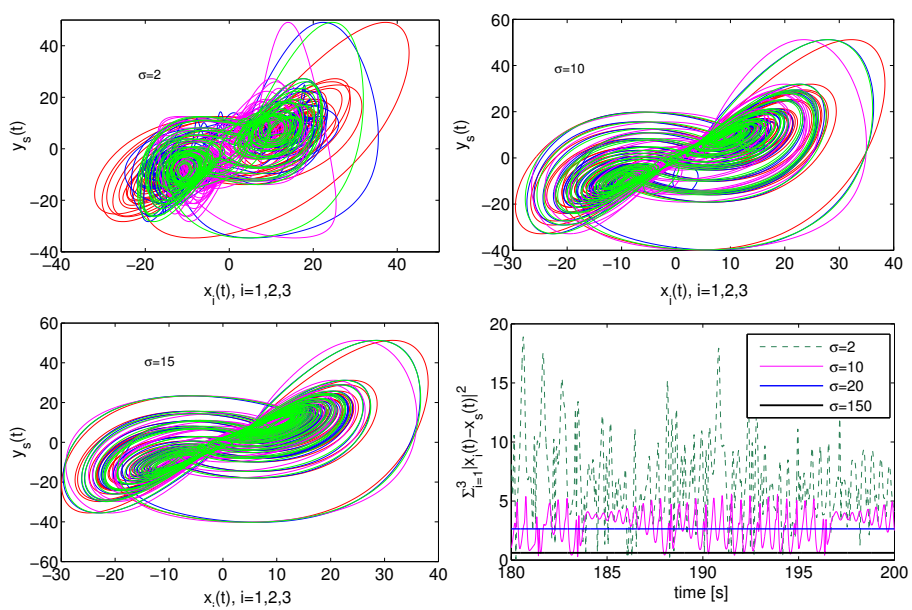

Fig. 4. First three plots: phase portraits of the three chaotic oscillators compared to that of the mean-field unit, for different values of the interconnection gain $\sigma$. In all the plots the ordinates axes refer to $y_{s}(t)$. The lower-right plot depicts the synchronization errors $\left|\boldsymbol{e}_{y}(t)\right|$.
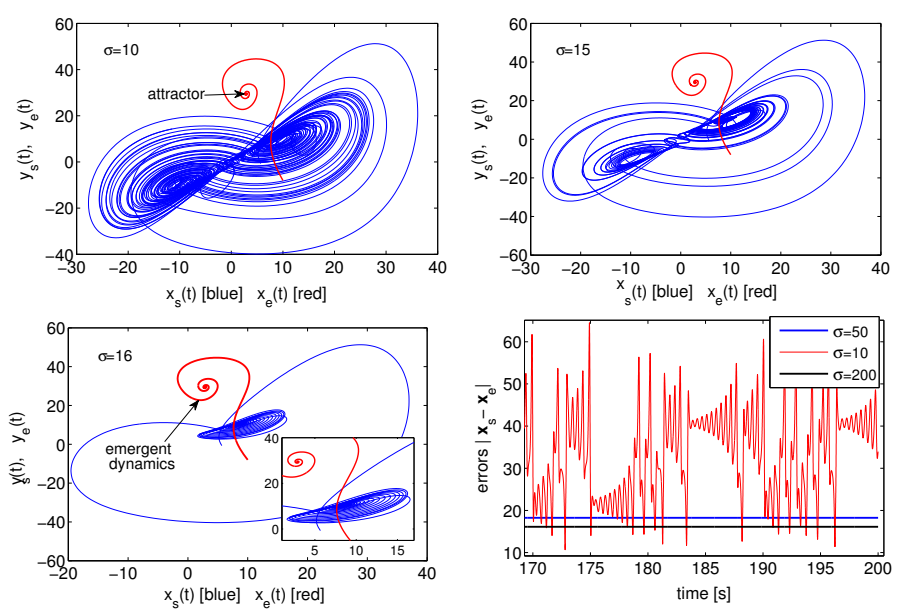

Fig. 5. $x-y$ phase portraits corresponding to the emergent dynamics (28) and the mean-field dynamics $(51 \mathrm{~b})$ for different values of the interconnection gain $\sigma$. Bottom-right: $\left|\boldsymbol{x}_{s}(t)\right|_{\mathcal{A}}$. 
Finally, the lower-right plot in Figure 5 depicts $\mid \boldsymbol{x}_{s}(t)-$ $\left.\boldsymbol{x}_{e}(t)|=| \boldsymbol{x}_{s}(t)\right|_{\mathcal{A}}$ which corresponds to the difference between the solutions $\boldsymbol{x}_{s}(t)$ of the mean-field system (51b) and $\boldsymbol{x}_{e}(t)$, solution of the emergent dynamics $\dot{\boldsymbol{x}}_{e}=f_{s}\left(\boldsymbol{x}_{e}\right)$. Note that this difference diminishes as the interconnection gain increases however, it does not vanish as $\sigma \rightarrow \infty$ since the synchronization errors do not converge to zero.

\section{CONCLUSIONS AND FURTHER RESEARCH}

We have set the basis of an analysis framework for networks of systems that are heterogeneous both in the dynamics and the parameters. This framework builds upon the original concept of dynamic consensus, introduced here, and that of emergent dynamics. Together, these are suitably tailored to study collective behavior of networked-interconnected systems with different models.

Our results, however, focus on networks under diffusive coupling and, to guarantee that the trajectories are ultimately bounded, we appeal to a semi-passivity assumption. Relaxing this hypothesis, for instance, in terms of input-to-state-stability, might allow to cope with networks under nonlinear coupling. From a systems viewpoint, many other important problems remain open. Firstly, to extend our framework to cover other classes of systems, such as hybrid systems or discrete-timebased. In addition, constrained by the nature of the diffusive coupling, we assume that the inputs and outputs have the same dimension. Extending our results to the case of systems with heterogeneous dimensions (of the state, the inputs and the outputs) is another well-motivated problem.

Last but not least, the problem of control design, based on the dichotomy exhibited here (considering the synchronisation and the mean field behaviour), is completely open. For instance, to what extent, for a given network, can one impose a target emergent dynamics? Note that this question may be posed independently of whether the nature of coupling is given or it constitutes an additional design degree of freedom.

\section{ACKNOWLEDGMENTS}

The authors greatly acknowledge the many technical comments of Nathan van de Wouw on previous versions of this material; his input was a guideline to prepare the presentation of our results.

This article is supported by Government of Russian Federation (grant 074-U01).

\section{REFERENCES}

[1] I. I. Blekhman, A. L. Fradkov, H. Nijmeijer, and A. Y. Pogromsky, "On self-synchronization and controlled synchronization," Systems and Control Letters, vol. 31, pp. 299-305, 1997.

[2] H. Haken, Synergetics. An Introduction. Nonequilibrium Phase Transitions and Self-Organization in Physics, Chemistry, and Biology. Series in Synergetics, Berlin: Springer-Verlag, 3rd ed., 1983.

[3] G. Nicolis and I. Prigogine, Self-organization in nonequilibrium systems. New York: John Wiley, 1st ed., 1977.

[4] H. Nijmeijer and A. Rodríguez-Angeles, Synchronization of mechanical systems, vol. 46 of Nonlinear Science, Series A. World Scientific, 2003.

[5] H. L. Trentelman, K. Takaba, and N. Monshizadeh, "Robust synchronization of uncertain linear multi-agent systems," IEEE Transactions on Automatic Control, vol. 58, pp. 1511-1523, June 2013.
[6] T. Liu, D. Hill, and J. Zhao, "Output synchronization of dynamical networks with incrementally-dissipative nodes and switching topology," IEEE Transactions on Circuits and Systems I: Fundamental Theory and Applications, vol. 62, no. 9, pp. 2312-2323, 2015.

[7] R. Olfati-Saber and R. Murray, "Consensus problems in networks of agents with switching topology and time-delays," Automatic Control, IEEE Transactions on, vol. 49, no. 9, pp. 1520 - 1533, 2004

[8] P. Wieland, J. Wu, and F. Allgöwer, "On synchronous steady states and internal models of diffusively coupled systems," IEEE Transactions on Automatic Control, vol. 58, no. 10, pp. 2591-2602, 2013.

[9] J. M. Montenbruck, M. Bürger, and F. Allgöwer, "Practical synchronization with diffusive couplings," Automatica, vol. 53, pp. 235-243, 2015.

[10] W. Ren and R. W. Beard, Distributed consensus in multivehicle cooperative control. Springer verlag, 2005.

[11] I. Belykh, E. de Lange, and M. Hasler, "Synchronization of bursting neurons: What matters in the network topology," Physics Review Letters, vol. 94, p. 188101, 2005. http://dx.doi.org/10.1103/PhysRevLett.94.188101.

[12] N. Corson, M. A. Aziz-Alaoui, R. Ghnemat, S. Balev, and C. Bertelle, "Modeling the dynamics of complex interaction systems: From morphogenesis to control," International Journal of Bifurcation and Chaos, vol. 22, p. "1250025 [20 pages]", 2012. DOI: $10.1142 / \mathrm{S} 0218127412500253$

[13] M. Mamat, P. W. Kurniawan, and A. Kartono, "Development of dynamics and synchronization model for coupled neurons using HindmarshRose model," Applied Mathematical Sciences, vol. 7, no. 3, pp. 135-152, 2013.

[14] T. Liu, J. Zhao, and D. J. Hill, "Synchronization of complex delayed dynamical networks with nonlinearly coupled nodes," Chaos, Solitons and Fractals, vol. 40, pp. 1506-1519, 2009.

[15] J. Y. Wang, J. W. Feng, C. Xu, and Y. Zhao, "Cluster synchronization of nonlinearly-coupled complex networks with nonidentical nodes and asymmetrical coupling matrix," Nonlinear Dynamics, vol. 67, no. 2, pp. 1635-1646, 2012.

[16] C. Altafini, "Dynamics of opinion forming in structurally balanced social networks," PloS one, vol. 7, no. 6, p. e38135, 2012.

[17] J. Xiang, Y. Li, and D. J. Hill, "Cooperative output regulation of multi-agent network systems with dynamic edges," arXiv preprint arXiv:1602.02598, 2016.

[18] G. Casadei, L. Marconi, and C. D. Persis, "Synchronization of nonlinear oscillators over networks with dynamic links," in Proceedings of the 54th IEEE Conference on Decision and Control, p. 2015.

[19] C. Godsil and G. F. Royle, Algebraic graph theory, vol. 207. Springer Science \& Business Media, 2013.

[20] W. Zhang, M. S. Branicky, and S. M. Phillips, "Stability of networked control systems," , IEEE Control Systems Magazine, vol. 21, no. 1, pp. 84-99, 2001.

[21] K. D. Kim and P. R. Kumar, "Cyber-physical systems: A perspective at the centennial," Proceedings of the IEEE, vol. 100, no. Special Centennial Issue, pp. 1287-1308, 2012.

[22] A. Y. Pogromski and H. Nijmeijer, "Cooperative oscillatory behavior of mutually coupled dynamical systems," IEEE Transactions on Circuits and Systems I: Fundamental Theory and Applications, vol. 48, no. 2, pp. 152-162, 2001.

[23] A. Y. Pogromski, T. Glad, and H. Nijmeijer, "On difffusion driven oscillations in coupled dynamical systems," International Journal of Bifurcation and Chaos in Applied Sciences and Engineering, vol. 9, no. 4, pp. 629-644, 1999.

[24] P. DeLellis, M. Di Bernardo, and D. Liuzza, "Convergence and synchronization in heterogeneous networks of smooth and piecewise smooth systems," Automatica, vol. 56, pp. 1-11, 2015.

[25] W. Ren and R. W. Beard, "Consensus seeking in multi-agent systes under dynamically changing interaction topologies," IEEE Transactions on Automatic Control, vol. 50, no. 5, pp. 655-661, 2005.

[26] A. Isidori, L. Marconi, and G. Casadei, "Robust output synchronization of a network of heterogeneous nonlinear agents via nonlinear regulation theory," IEEE Transactions on Automatic Control, vol. 59, no. 10, pp. 2680-2691, 2014.

[27] L. Zhu, Z. Chen, and R. H. Middleton, "A general framework for robust output synchronization of heterogeneous nonlinear networked systems," IEEE Transactions on Automatic Control, vol. 61, no. 8, pp. 2092-2107, 2016. DOI: 10.1109/TAC.2015.2492141.

[28] L. Conteville and E. Panteley, "Practical synchronization in complex networks of nonidentical dynamical nodes," in 20th International Symposium on Mathematical Theory of Networks and Systems, (Melbourne, $\mathrm{AU}), 2012$. 
[29] E. Panteley, L. Conteville, and A. Loría, "On practical synchronization of a network of diffusively coupled hindmarsh-rose neuron," in European Nonlinear Oscillations Conference, (Wien, Austria), 2014.

[30] E. Panteley, A. Loria, and L. Conteville, "On practical synchronization of heterogeneous networks of nonlinear systems," in Proc. IEEE American Control Conference, (Chicago, IL, USA), pp. 5359-5364, 2015.

[31] E. Panteley, "A stability-theory perspective to synchronisation of heterogeneous networks." Habilitation à diriger des recherches (DrSc dissertation). Université Paris Sud, Orsay, France, 2015.

[32] A. Isidori, Nonlinear control systems II. Springer Verlag: London, 1999.

[33] H. Khalil, Nonlinear systems. New York: Prentice Hall, 3rd ed., 2002.

[34] A. Y. Pogromski, G. Santoboni, and H. Nijmeijer, "Partial synchronization: from symmetry towards stability," Physica $D$ : Nonlinear Phenomena, vol. 172, no. 1-4, pp. 65-87, 2002.

[35] T. Oguchi, T. Yamamoto, and H. Nijmeijer, Topics in Time Delay Systems, ch. Synchronization of bidirectionally coupled nonlinear systems with time-varying delay, pp. 391-401. Springer Berlin:Heidelberg, 2009.

[36] Y. C. Liu and N. Chopra, "Robust controlled synchronization of interconnected robotic systems," in Proc. IEEE American Control Conference, pp. 1434-1439, 2010.

[37] D. Angeli, "A Lyapunov approach to incremental stability properties," Automatic Control, IEEE Transactions on, vol. 47, pp. 410-421, Mar 2002.

[38] W. Ren, R. W. Beard, and T. W. McLain, Coordination variables and consensus building in multiple vehicle systems, vol. 309 of Lecture Notes in Control and Information Sciences, ch. in Cooperative Control, pp. 171-188. V. Kumar, N. E. Leonard, and A. S. Morse, eds., Berlin: Springer-Verlag, 2005.

[39] C. W. Wu, "Synchronization in networks of nonlinear dynamical systems coupled via a directed graph," Nonlinearity, vol. 18, no. 3, p. 1057, 2005.

[40] J. M. Ortega, Matrix theory: A second course. Springer Science \& Business Media, 2013.

[41] A. R. Teel, J. Peuteman, and D. Aeyels, "Semi-global practical asymptotic stability and averaging," Systems and Control Letters, vol. 37, no. 5 pp. 329-334, 1999.

[42] A. Chaillet and A. Loria, "Necessary and sufficient conditions for uniform practical asymptotic stability: application to cascaded systems," Automatica, vol. 42, no. 11, pp. 1899-1906, 2006.

[43] M. Corless and G. Leitmann, "Continuous state feedback guaranteeing uniform ultimate boundedness for uncertain dynamic systems," IEEE Transactions on Automatic Control, vol. 26, no. 5, pp. 1139-1144, 1981.

[44] T. Yoshizawa, Stability theory by Lyapunov's second method. Tokyo: The Mathematical Society of Japan, 1966.

[45] E. Steur, I. Tyukin, and H. Nijmeijer, "Semi-passivity and synchronization of diffusively coupled neuronal oscillators," Physica D: Nonlinear Phenomena, vol. 238, no. 21, pp. 2119-2128, 2009.

[46] T. Yoshizawa, Stability theory by Lyapunov's second method. Tokyo: The Mathematical Society of Japan, 1966.

[47] E. N. Lorenz, "Deterministic nonperiodic flow," J. Atmos. Sci., vol. 20, pp. 130-141, 1963.

[48] J. H. Lü and G. Chen, "A new chaotic attractor coined," International Journal of Bifurcation and Chaos, pp. 659-661, 2002.

[49] A. Teel, E. Panteley, and A. Loría, "Integral characterizations of uniform asymptotic and exponential stability with applications," Math. of Cont. Sign. and Syst., vol. 15, pp. 177-201, 2002.

\section{APPENDIX}

\section{A. Proof of Lemma 1}

By assumption, $L \in \mathbb{R}^{N \times N}$ has one zero eigen-value $\lambda_{1}(L)=0$ with associated right eigenvector $v_{r 1} \in \mathbb{R}^{N}$, left eigen-vector $v_{\ell 1} \in \mathbb{R}^{N}$, and $M \leq N-1$ eigen-values $\lambda_{i}(L) \in \mathbb{C}$ with multiplicity $\mu_{i} \geq 1$ such that $\Re e\left(\lambda_{i}\right)>0$.

The matrix $L$ admits the Jordan decomposition $L=$ $U \Lambda U^{-1}$ where $U \in \mathbb{C}^{N \times N}$ is non-singular and $\Lambda:=$ blockdiag $\left[\Lambda_{i}\right]$ with $\Lambda_{1}=0$-see Eq. (13). Similarly to Eq. (14), we partition $U:=\left[\begin{array}{ll}v_{r 1} & U_{1}\end{array}\right]$. Next, define $\Lambda_{R_{i}}:=\Re e\left(\Lambda_{i}\right)$ for all $i \in[2, M]$. Since $\Re e\left(\lambda_{i}\right)>0$ for each $i \in[2, M]$ there exists a matrix $P_{i} \in \mathbb{R}^{\mu_{i} \times \mu_{i}}$, symmetric and positive definite, such that $P_{i} \Lambda_{R_{i}}+\Lambda_{R_{i}}^{\top} P_{i}=I$. It follows from this that for any $z \in \mathbb{R}^{\mu_{i}}, z^{\top}\left(P_{i} \Lambda_{i}+\Lambda_{i}^{*} P_{i}\right) z=|z|^{2}$. To see this, note that

$$
P_{i} \Lambda_{i}+\Lambda_{i}^{*} P_{i}=P_{i}\left(\Lambda_{R_{i}}+j \Lambda_{I_{i}}\right)+\left(\Lambda_{R_{i}}-j \Lambda_{I_{i}}\right)^{\top} P_{i}
$$

hence

$$
\begin{aligned}
z^{\top}\left[P_{i} \Lambda_{i}\right. & \left.+\Lambda_{i}^{*} P_{i}\right] z \\
& =z^{\top}\left[P_{i}\left(\Lambda_{R_{i}}+j \Lambda_{I_{i}}\right)+\left(\Lambda_{R_{i}}-j \Lambda_{I_{i}}\right)^{\top} P_{i}\right] z \\
& =z^{\top}\left[P_{i} \Lambda_{R_{i}}+\Lambda_{R_{i}}^{\top} P_{i}\right] z .
\end{aligned}
$$

Next, let us introduce the block-diagonal matrix $\mathcal{P} \in \mathbb{R}^{N \times N}$

$$
\mathcal{P}:=\left[\begin{array}{cccc}
1 & 0 & \cdots & 0 \\
0 & P_{2} & & \vdots \\
\vdots & & \ddots & 0 \\
0 & \cdots & 0 & P_{M}
\end{array}\right]
$$

which is positive definite since so are $P_{i}$ for each $i \in[2, M]$. Note that, moreover,

$$
\boldsymbol{x}^{\top}\left[\mathcal{P} \Lambda+\Lambda^{*} \mathcal{P}\right] \boldsymbol{x}=\boldsymbol{x}^{\top} J_{0} \boldsymbol{x}, \quad J_{0}:=\left[\begin{array}{cc}
0 & \cdots \\
\vdots & I_{N-1}
\end{array}\right] .
$$

Then, let $\Gamma_{c}:=\left(U^{-1}\right)^{*} \mathcal{P} U^{-1}$. This matrix is Hermitian positive definite since $\mathcal{P}$ is symmetric positive definite and $U^{-1}$ has full rank by definition. From the previous computations, it follows that $\boldsymbol{e}^{\top} \Gamma_{c} \boldsymbol{e}=\boldsymbol{e}^{\top} \Re e\left(\Gamma_{c}\right) \boldsymbol{e}$ hence, defining $\Gamma:=\Re e\left(\Gamma_{c}\right)$, we see that $V$ in (23) is positive definite and proper and takes values in $\mathbb{R}$.

Next, we differentiate $V$. To that end, note that, in view of (22) and the property that $L v_{r 1}=0, \dot{\boldsymbol{x}}=-L \boldsymbol{x}$ is equivalent to $\dot{e}=-L e$. Therefore, evaluating the total derivative of $V$ we obtain

$$
\begin{aligned}
\dot{V}(\boldsymbol{e}) & =-\boldsymbol{e}^{\top} \Gamma L \boldsymbol{e}-\boldsymbol{e}^{\top} L^{\top} \Gamma \boldsymbol{e} \\
& =-\boldsymbol{e}^{\top} \Gamma_{c} L \boldsymbol{e}-\boldsymbol{e}^{\top} L^{\top} \Gamma_{c}^{*} \boldsymbol{e} \\
& =-\boldsymbol{e}^{\top}\left(U^{-1}\right)^{*} \mathcal{P} U^{-1} L \boldsymbol{e}-\boldsymbol{e}^{\top} L^{\top}\left(U^{-1}\right)^{*} \mathcal{P} U^{-1} \boldsymbol{e} \\
& =-\boldsymbol{e}^{\top}\left(U^{-1}\right)^{*} \mathcal{P} U^{-1} U \Lambda U^{-1} \boldsymbol{e} \\
& \quad-\boldsymbol{e}^{\top}\left(U^{-1}\right)^{*} \Lambda^{*} U^{*}\left(U^{-1}\right)^{*} \mathcal{P} U^{-1} \boldsymbol{e} \\
= & -\boldsymbol{e}^{\top}\left(U^{-1}\right)^{*}\left[\mathcal{P} \Lambda+\Lambda^{*} \mathcal{P}\right] U^{-1} \boldsymbol{e} \\
= & -\boldsymbol{e}^{\top}\left(U^{-1}\right)^{*} J_{0} U^{-1} \boldsymbol{e} .
\end{aligned}
$$

Furthermore, we have $e^{\top}\left(U^{-1}\right)^{*}=\left(U^{-1} \boldsymbol{e}\right)^{*}$ and

$$
U^{-1} e=:\left[\begin{array}{l}
0 \\
\xi
\end{array}\right]
$$

where $\boldsymbol{\xi} \in \mathbb{C}^{N-1}$. Hence,

$$
\dot{V}(\boldsymbol{e})=-\boldsymbol{\xi}^{*} \boldsymbol{\xi} \leq-\left(1 / c_{U}\right)^{2}|\boldsymbol{e}|^{2}
$$

where we defined $c_{U}:=\left|U_{1}\right|$ and we used the fact that $e=$ $U_{1} \boldsymbol{\xi}$. The statement follows with $\gamma:=\left(1 / c_{U}\right)^{2}$.

\section{B. Proof of Proposition 1}

Let us introduce the set

$$
\mathcal{B}:=\left\{\boldsymbol{x} \in \mathbb{R}^{n}:|\boldsymbol{x}|_{\mathcal{A}} \leq \alpha_{3}^{-1}(c / \eta) \cdot\right\}
$$

Then, we have

$$
|\boldsymbol{x}|_{\mathcal{B}}=\left\{\begin{array}{cl}
|\boldsymbol{x}|_{\mathcal{A}}-\alpha_{3}^{-1}(c / \eta) & \text { if }|\boldsymbol{x}|_{\mathcal{A}}>\alpha_{3}^{-1}(c / \eta) \\
0 & \text { otherwise. }
\end{array}\right.
$$


Then, let us introduce the locally Lipschitz function $W$ : $\mathbb{R}^{n} \rightarrow \mathbb{R}$ such that

$$
W(\boldsymbol{x}):=\max \left\{0, V(\boldsymbol{x})-V\left(\alpha_{3}^{-1}(c / \eta)\right)\right\} .
$$

Note that $W$ is positive definite, radially unbounded and decrescent with respect to $\mathcal{B}$ that is, $W(\boldsymbol{x})=0$ for all $\boldsymbol{x} \in \mathcal{B}$, $W(\boldsymbol{x})>0$ for all $\boldsymbol{x} \notin \mathcal{B}$, and $W(\boldsymbol{x}) \rightarrow \infty$ as $|\boldsymbol{x}|_{\mathcal{B}} \rightarrow \infty$. This follows from (48) as well as the compactness of $\mathcal{B}$ and the continuity of $W$. As a matter of fact, there exist $\alpha_{1}^{\prime}$ and $\alpha_{2}^{\prime} \in \mathcal{K}_{\infty}$ such that, for all $\boldsymbol{x} \in \mathbb{R}^{n}$,

$$
\alpha_{1}^{\prime}\left(|\boldsymbol{x}|_{\mathcal{B}}\right) \leq W(\boldsymbol{x}) \leq \alpha_{2}^{\prime}\left(|\boldsymbol{x}|_{\mathcal{B}}\right)
$$

Furthermore,

$$
\dot{W}(\boldsymbol{x}) \leq \max \left\{0,-\eta \alpha_{3}(|\boldsymbol{x}|)+c\right\}
$$

hence, we have $\dot{W}(\boldsymbol{x})=0$ for all $\boldsymbol{x} \in \mathcal{B}$ and, since $\eta>1$ and $\alpha_{3} \in \mathcal{K}_{\infty}$, we have $\dot{W}(\boldsymbol{x})<0$ for all $\boldsymbol{x} \notin \mathcal{B}$. Therefore, one we may construct a continuous function $\alpha_{3}^{\prime} \in \mathcal{K}_{\infty}$ such that

$$
\dot{W}(\boldsymbol{x}) \leq-\alpha_{3}^{\prime}\left(|x|_{\mathcal{B}}\right)
$$

for all $\boldsymbol{x} \in \mathbb{R}^{n}$. Next, we integrate along the trajectories, on both sides of the latter inequality and we use (78) to obtain

$$
\int_{0}^{\infty} \alpha_{3}^{\prime}\left(|x(t)|_{\mathcal{B}}\right) d t \leq \alpha_{2}^{\prime}\left(|x(0)|_{\mathcal{B}}\right) .
$$

Then, invoking [49, Theorem 1] we conclude that there exists a class $\mathcal{K} \mathcal{L}$ function $\beta$ such that

$$
\left|x\left(t, \boldsymbol{x}_{\circ}\right)\right|_{\mathcal{B}} \leq \beta\left(\left|x_{\circ}\right|_{\mathcal{B}}, t\right)
$$

which, since $|\boldsymbol{x}|_{\mathcal{B}} \leq|\boldsymbol{x}|_{\mathcal{A}} \leq|\boldsymbol{x}|_{\mathcal{B}}+\alpha_{3}^{-1}(c / \eta)$, implies that

$$
\begin{aligned}
\left|\boldsymbol{x}\left(t, \boldsymbol{x}_{\circ}\right)\right|_{\mathcal{A}} & \leq\left|\boldsymbol{x}\left(t, \boldsymbol{x}_{\circ}\right)\right|_{\mathcal{B}}+\alpha_{3}^{-1}(c / \eta) \\
& \leq \beta\left(\left|\boldsymbol{x}_{\circ}\right|_{\mathcal{B}}, t\right)+\alpha_{3}^{-1}(c / \eta) \\
& \leq \beta\left(\left|\boldsymbol{x}_{\circ}\right|_{\mathcal{A}}, t\right)+\alpha_{3}^{-1}(c / \eta) .
\end{aligned}
$$

It is clear that $\delta(\eta):=\alpha_{3}^{-1}(c / \eta)$ tends to zero as $\eta \rightarrow \infty$.

\section{Technical lemmas}

Lemma 2 Let $s_{i}$, with $i \in \mathcal{I}$ be arbitrary non-negative scalars, then for any class $\mathcal{K}_{\infty}$ function $\alpha(\cdot)$, we have

$$
\alpha\left(\frac{1}{N} \sum_{i=1}^{N} s_{i}\right) \leq \sum_{i=1}^{N} \alpha\left(s_{i}\right) \leq N \alpha\left(\sum_{i=1}^{N} s_{i}\right)
$$

Proof: Let $s_{k}=\max _{i=1, \ldots, N}\left\{s_{i}\right\}$, then we have that $\frac{1}{N} \sum_{i=1}^{N} s_{i} \leq s_{k}$ and therefore

$$
\alpha\left(\frac{1}{N} \sum_{i=1}^{N} s_{i}\right) \leq \alpha\left(s_{k}\right)
$$

so the first inequality holds. Similarly, for the second inequality we have

$$
\sum_{i=1}^{N} \alpha\left(s_{i}\right) \leq N \alpha\left(s_{k}\right)
$$

Lemma 3 Let $\boldsymbol{x}_{1}, \ldots, \boldsymbol{x}_{N} \in \mathbb{R}^{n}, \boldsymbol{x}=\left[\boldsymbol{x}_{1}^{\top}, \boldsymbol{x}_{2}^{\top}, \ldots, \boldsymbol{x}_{N}^{\top}\right]^{\top} \in$ $\mathbb{R}^{n N}, v_{\ell 1}=\left[\nu_{1}, \cdots, \nu_{N}\right]^{\top}$, and $\boldsymbol{x}_{s}=\sum_{i=1}^{N} \nu_{i} \boldsymbol{x}_{i}$ with $\nu_{i}>0$ for all $i \leq N$. Then,

$$
\begin{gathered}
\left|\boldsymbol{x}-\mathbf{1}_{N} \otimes \boldsymbol{x}_{s}\right|^{2} \geq \frac{1}{2 N} \sum_{i=1}^{N} \sum_{j=1}^{N}\left|\boldsymbol{x}_{i}-\boldsymbol{x}_{j}\right|^{2}, \\
\sum_{i=1}^{N} \sum_{j=1}^{N}\left|\boldsymbol{x}_{i}-\boldsymbol{x}_{j}\right|^{2} \geq \frac{\left|\boldsymbol{x}-\mathbf{1}_{N} \otimes \boldsymbol{x}_{s}\right|^{2}}{\left|v_{\ell 1} v_{\ell 1}^{\top}\right|_{2}} .
\end{gathered}
$$

Proof: Let us define the vectors $\boldsymbol{X}, \tilde{\boldsymbol{X}} \in \mathbb{R}^{n N^{2}}$,

$$
\boldsymbol{X}=\mathbf{1}_{N} \otimes \boldsymbol{x}=\left[\begin{array}{c}
\boldsymbol{x} \\
\vdots \\
\boldsymbol{x}
\end{array}\right], \quad \tilde{\boldsymbol{X}}=\left[\begin{array}{c}
\boldsymbol{x}-\mathbf{1}_{N} \otimes \boldsymbol{x}_{1} \\
\vdots \\
\boldsymbol{x}-\mathbf{1}_{N} \otimes \boldsymbol{x}_{N}
\end{array}\right] .
$$

In order to prove that Inequality (80) holds, we show that

$$
\begin{gathered}
\tilde{\boldsymbol{X}}^{\top} \tilde{\boldsymbol{X}}=\sum_{i=1}^{N} \sum_{j=1}^{N}\left|\boldsymbol{x}_{i}-\boldsymbol{x}_{j}\right|^{2} \\
\tilde{\boldsymbol{X}}^{\top} \tilde{\boldsymbol{X}} \leq 2 N\left|\boldsymbol{x}-\mathbf{1}_{N} \otimes \boldsymbol{x}_{s}\right|^{2} .
\end{gathered}
$$

Concerning the former, we see that, by definition,

$$
\tilde{\boldsymbol{X}}^{\top} \tilde{\boldsymbol{X}}=\sum_{i=1}^{N}\left|\boldsymbol{x}-\mathbf{1}_{N} \otimes \boldsymbol{x}_{i}\right|^{2},
$$

and, by direct computation, we see that, for each $i \leq N$,

$$
\left[\boldsymbol{x}-\mathbf{1}_{N} \otimes \boldsymbol{x}_{i}\right]^{\top}\left[\boldsymbol{x}-\mathbf{1}_{N} \otimes \boldsymbol{x}_{i}\right]=\sum_{k=1}^{N}\left|\boldsymbol{x}_{k}-\boldsymbol{x}_{i}\right|^{2},
$$

from which (83) follows.

Next, to establish (83) we observe that

$$
\begin{aligned}
\tilde{\boldsymbol{X}}= & \mathbf{1}_{N} \otimes \boldsymbol{x}-\left[\begin{array}{c}
\mathbf{1}_{N} \otimes \boldsymbol{x}_{1} \\
\vdots \\
\mathbf{1}_{N} \otimes \boldsymbol{x}_{N}
\end{array}\right] \\
= & \mathbf{1}_{N} \otimes\left[\boldsymbol{x}-\mathbf{1}_{N} \otimes \boldsymbol{x}_{s}\right]+\mathbf{1}_{N} \otimes\left[\mathbf{1}_{N} \otimes \boldsymbol{x}_{s}\right] \\
& -\left[\begin{array}{c}
\mathbf{1}_{N} \otimes\left[\left(\boldsymbol{x}_{1}-\boldsymbol{x}_{s}\right)+\boldsymbol{x}_{s}\right] \\
\vdots \\
\mathbf{1}_{N} \otimes\left[\left(\boldsymbol{x}_{N}-\boldsymbol{x}_{s}\right)+\boldsymbol{x}_{s}\right]
\end{array}\right] \\
= & \mathbf{1}_{N} \otimes \boldsymbol{e}+\left[\mathbf{1}_{N} \otimes \mathbf{1}_{N}\right] \otimes \boldsymbol{x}_{s} \\
& -\left[\begin{array}{c}
\left.\mathbf{1}_{N} \otimes \boldsymbol{e}_{1}\right] \\
\vdots \\
\mathbf{1}_{N} \otimes \boldsymbol{e}_{N}
\end{array}\right]-\left[\begin{array}{c}
\mathbf{1}_{N} \otimes \boldsymbol{x}_{s} \\
\vdots \\
\mathbf{1}_{N} \otimes \boldsymbol{x}_{s}
\end{array}\right] \\
= & \mathbf{1}_{N} \otimes \boldsymbol{e}-\boldsymbol{E}
\end{aligned}
$$

where we recall that $\boldsymbol{e}=\boldsymbol{x}-\mathbf{1}_{N} \otimes \boldsymbol{x}_{s}$ and we defined

$$
\boldsymbol{E}:=\left[\begin{array}{c}
\mathbf{1}_{N} \otimes \boldsymbol{e}_{1} \\
\vdots \\
\mathbf{1}_{N} \otimes \boldsymbol{e}_{N}
\end{array}\right]
$$

It follows that

$$
\tilde{\boldsymbol{X}}^{\top} \tilde{\boldsymbol{X}}=\left[\mathbf{1}_{N} \otimes \boldsymbol{e}-\boldsymbol{E}\right]^{\top}\left[\mathbf{1}_{N} \otimes \boldsymbol{e}-\boldsymbol{E}\right]
$$




$$
\begin{aligned}
& =\left[\mathbf{1}_{N}^{\top} \otimes \boldsymbol{e}^{\top}-\boldsymbol{E}^{\top}\right]\left[\mathbf{1}_{N} \otimes \boldsymbol{e}-\boldsymbol{E}\right] \\
& =\mathbf{1}_{N}^{\top} \mathbf{1}_{N} \otimes \boldsymbol{e}^{\top} \boldsymbol{e}+\boldsymbol{E}^{\top} \boldsymbol{E}-2 \boldsymbol{E}^{\top}\left[\mathbf{1}_{N} \otimes \boldsymbol{e}\right] \\
& =N \boldsymbol{e}^{\top} \boldsymbol{e}+\boldsymbol{E}^{\top} \boldsymbol{E}-2 \boldsymbol{E}^{\top}\left[\mathbf{1}_{N} \otimes \boldsymbol{e}\right]
\end{aligned}
$$

Now, on one hand, we have

$$
\begin{aligned}
\boldsymbol{E}^{\top} \boldsymbol{E} & =\left[\mathbf{1}_{N}^{\top} \otimes \boldsymbol{e}_{1}^{\top}, \cdots, \mathbf{1}_{N}^{\top} \otimes \boldsymbol{e}_{N}^{\top}\right]\left[\begin{array}{c}
\mathbf{1}_{N} \otimes \boldsymbol{e}_{1} \\
\vdots \\
\mathbf{1}_{N}^{\top} \otimes \boldsymbol{e}_{N}
\end{array}\right] \\
& =\sum_{k=1}^{N}\left[\mathbf{1}_{N}^{\top} \mathbf{1}_{N}\right] \otimes\left[\boldsymbol{e}_{k}^{\top} \boldsymbol{e}_{k}\right] \\
& =N \sum_{k=1}^{N} \boldsymbol{e}_{k}^{\top} \boldsymbol{e}_{k}=N \boldsymbol{e}^{\top} \boldsymbol{e} .
\end{aligned}
$$

On the other hand,

$$
\begin{aligned}
\boldsymbol{E}^{\top}\left[\mathbf{1}_{N} \otimes \boldsymbol{e}\right] & =\left[\mathbf{1}_{N}^{\top} \otimes \boldsymbol{e}_{1}^{\top}, \cdots, \mathbf{1}_{N}^{\top} \otimes \boldsymbol{e}_{N}^{\top}\right]\left[\begin{array}{c}
\boldsymbol{e} \\
\vdots \\
\boldsymbol{e}
\end{array}\right] \\
& =\left[\boldsymbol{e}^{\top}, \cdots, \boldsymbol{e}^{\top}\right]\left[\begin{array}{c}
\mathbf{1}_{N} \otimes \boldsymbol{e}_{1} \\
\vdots \\
\mathbf{1}_{N} \otimes \boldsymbol{e}_{N}
\end{array}\right] \\
& =\sum_{k=1}^{N} \boldsymbol{e}^{\top}\left[\mathbf{1}_{N} \otimes \boldsymbol{e}_{k}\right]=\boldsymbol{e}^{\top}\left[\mathbf{1}_{N} \otimes \sum_{k=1}^{N} \boldsymbol{e}_{k}\right] .
\end{aligned}
$$

Furthermore, using the identity

$$
\sum_{k=1}^{N} \boldsymbol{e}_{k}=\sum_{k=1}^{N} \boldsymbol{x}_{k}-N \boldsymbol{x}_{s}
$$

in (87) we obtain,

$$
\begin{aligned}
& \boldsymbol{e}^{\top}\left[\mathbf{1}_{N} \otimes \sum_{k=1}^{N} \boldsymbol{e}_{k}\right]=\boldsymbol{e}^{\top}\left[\begin{array}{c}
\sum_{k=1}^{N} \boldsymbol{x}_{k}-N \boldsymbol{x}_{s} \\
\vdots \\
\sum_{k=1}^{N} \boldsymbol{x}_{k}-N \boldsymbol{x}_{s}
\end{array}\right] \\
& =\left[\boldsymbol{x}_{1}^{\top}-\boldsymbol{x}_{s}^{\top}, \cdots, \boldsymbol{x}_{N}-\boldsymbol{x}_{s}^{\top}\right]\left[\begin{array}{c}
\sum_{k=1}^{N} \boldsymbol{x}_{k}-N \boldsymbol{x}_{s} \\
\vdots \\
\sum_{k=1}^{N} \boldsymbol{x}_{k}-N \boldsymbol{x}_{s}
\end{array}\right] \\
& =\left[\sum_{k=1}^{N} \boldsymbol{x}_{k}-N \boldsymbol{x}_{s}\right]\left[\sum_{j=1}^{N} \boldsymbol{x}_{j}-N \boldsymbol{x}_{s}\right] .
\end{aligned}
$$

Using the latter, (87), and (86), in (85), we obtain

$$
\tilde{\boldsymbol{X}}^{\top} \tilde{\boldsymbol{X}}=2 N \boldsymbol{e}^{\top} \boldsymbol{e}-2\left[\sum_{k=1}^{N} \boldsymbol{x}_{k}-N \boldsymbol{x}_{s}\right]^{2} .
$$

From this and the identity $\boldsymbol{e}^{\top} \boldsymbol{e}=\left|\boldsymbol{x}-\mathbf{1}_{N} \otimes \boldsymbol{x}_{s}\right|^{2}$, (84) follows.

It is left to establish that (81) holds. To that end, we remark that

$$
\boldsymbol{e}=\left[\boldsymbol{x}-\mathbf{1}_{N} \otimes\left(v_{\ell 1}^{\top} \otimes I_{n}\right) \boldsymbol{x}\right]=\left[\begin{array}{c}
\boldsymbol{x}_{1}-\sum_{i=1}^{N} \nu_{i} \boldsymbol{x}_{i} \\
\vdots \\
\boldsymbol{x}_{N}-\sum_{i=1}^{N} \nu_{i} \boldsymbol{x}_{i}
\end{array}\right]
$$

which, by virtue of the identity $\mathbf{1}_{N} v_{\ell 1}=1$, i.e., $\sum_{i=1} a_{1}=1$, equals to

$$
\begin{aligned}
& {\left[\begin{array}{c}
{\left[\sum_{i=1}^{N} \nu_{i}\right] \boldsymbol{x}_{1}-\sum_{i=1}^{N} \nu_{i} \boldsymbol{x}_{i}} \\
\vdots \\
{\left[\sum_{i=1}^{N} \nu_{i}\right] \boldsymbol{x}_{N}-\sum_{i=1}^{N} \nu_{i} \boldsymbol{x}_{i}}
\end{array}\right]=-\left[\begin{array}{c}
\sum_{i=1}^{N} \nu_{i}\left[\boldsymbol{x}_{i}-\boldsymbol{x}_{1}\right] \\
\vdots \\
\sum_{i=1}^{N} \nu_{i}\left[\boldsymbol{x}_{i}-\boldsymbol{x}_{N}\right]
\end{array}\right]} \\
& =-\left[\begin{array}{c}
\left(v_{\ell 1}^{\top} \otimes I_{n}\right)\left[\boldsymbol{x}-\mathbf{1}_{N} \otimes \boldsymbol{x}_{1}\right] \\
\vdots \\
\left(v_{\ell 1}^{\top} \otimes I_{n}\right)\left[\boldsymbol{x}-\mathbf{1}_{N} \otimes \boldsymbol{x}_{N}\right]
\end{array}\right] \\
& =-\left[\begin{array}{ccc}
v_{\ell 1}^{\top} \otimes I_{n} & & 0 \\
& \ddots & \\
0 & & v_{\ell 1}^{\top} \otimes I_{n}
\end{array}\right]\left[\begin{array}{c}
\boldsymbol{x}-\mathbf{1}_{N} \otimes \boldsymbol{x}_{1} \\
\vdots \\
\boldsymbol{x}-\mathbf{1}_{N} \otimes \boldsymbol{x}_{N}
\end{array}\right]=D \tilde{\boldsymbol{X}}
\end{aligned}
$$

where we defined $D \in \mathbb{R}^{n N^{2}}$ as

$$
D:=\left[I_{N} \otimes\left(v_{\ell 1}^{\top} \otimes I_{n}\right)\right] \text {. }
$$

Thus, $\boldsymbol{e}^{\top} \boldsymbol{e}=\tilde{\boldsymbol{X}}^{\top} D^{\top} D \tilde{\boldsymbol{X}}$ and, therefore, $|\boldsymbol{e}|^{2} \leq\left|D^{\top} D\right|_{2}$. $|\tilde{\boldsymbol{X}}|$.

Next, we develop the product $D^{\top} D$. We have

$$
\begin{aligned}
& {\left[I_{N} \otimes\left(v_{\ell 1} \otimes I_{n}\right)\right]\left[I_{N} \otimes\left(v_{\ell 1}^{\top} \otimes I_{n}\right)\right]=} \\
& \quad\left[I_{N} \otimes\left(v_{\ell 1} \otimes I_{n}\right)\left(v_{\ell 1}^{\top} \otimes I_{n}\right)\right]=I_{N} \otimes\left[\left(v_{\ell 1} v_{\ell 1}^{\top}\right) \otimes I_{n}\right] .
\end{aligned}
$$

Then, using the identity $|A \otimes B|_{2}=|A|_{2} \cdot|B|_{2}$, which holds for any quadratic matrices $A$ and $B$, we see that, $\left|D^{\top} D\right|_{2}=$ $\left|I_{N}\right|_{2} \cdot\left|v_{\ell 1} v_{\ell 1}^{\top}\right|_{2} \cdot\left|I_{n}\right|_{2}$. We conclude that

$$
\tilde{\boldsymbol{X}}^{\top} \tilde{\boldsymbol{X}} \geq \frac{\boldsymbol{e}^{\top} \boldsymbol{e}}{\left|v_{\ell 1} v_{\ell 1}^{\top}\right|_{2}}
$$

which is equivalent to (81).

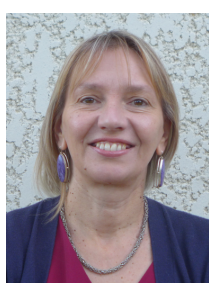

Elena Panteley received the M.Sc. and Ph.D. degrees in applied mathematics from the State University of St. Petersburg, St. Petersburg, Russia, in 1986 and 1997, respectively. From 1986 to 1998, she held a research position with the Institute for Problem of Mechanical Engineering, Russian Academy of Science, St. Petersburg. Since 2004 she holds a tenure position as Senior Researcher of the French National Centre of Scientific Research (CNRS), at the Laboratoire de signaux et systèmes, France. She is also associate researcher of ITMO University, St Petersbourg Russia, since 2014. Her research interests include stability and control of nonlinear dynamical systems, network systems with applications to electromechanical and neuronal systems.

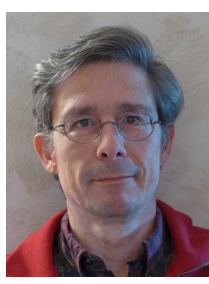

Antonio Loría was born in Mexico City in 1969. He got the BSc degree in Electronic Engineering from the ITESM, Monterrey, Mexico in 1991. He got the MSc and $\mathrm{PhD}$ degrees in Control Engg. from the UTC, France in 1993 and 1996 respectively. He is IEEE member since 1990. From December 1996 through Dec. 1998, he was successively an associate researcher at Univ. of Twente, The Netherlands; NTNU, Norway and the CCEC of the Univ. of California at Santa Barbara, CA, USA. A. Loria has the honour of holding a research position at the French National Centre of Scientific Research (CNRS) since january 1999 (as Senior Researcher since 2006). His research interests include dynamical systems theory (stability, chaos), control systems theory (adaptive, output feedback, discrete-time, continuous-time, linear, non-linear, hybrid, robust, passive, etc.). Between 2003-2015 he served as associate editor for Systems and Control Letters, Automatica, IEEE Transactions on Automatic Control, IEEE Transactions on Control Systems Technology, Control Systems Magazine and Revista Iberoamericana de Automatica Industrial. He has also been a member of the IEEE CSS Conference Editorial Board and diverse conference IPCs for several years. To see further, please visit http://antonio.loria.12s.supelec.fr. 